\title{
Small molecule epigenetic screen identifies novel EZH2 and HDAC inhibitors that target glioblastoma brain tumor-initiating cells
}

\author{
Natalie Grinshtein ${ }^{1}$, Constanza C. Rioseco${ }^{1}$, Richard Marcellus², David Uehling ${ }^{2}$, \\ Ahmed Aman ${ }^{2}$, Xueqing Lun ${ }^{3}$, Osamu Muto ${ }^{1}$, Lauren Podmore ${ }^{1}$, Jake Lever ${ }^{4}$, Yaoqing \\ Shen $^{4}$, Michael D. Blough ${ }^{3}$, Greg J. Cairncross ${ }^{3}$, Stephen M. Robbins ${ }^{3}$, Steven J. \\ Jones $^{4,5,6}$, Marco A. Marra ${ }^{4,5}$, Rima Al-Awar ${ }^{2}$, Donna L. Senger ${ }^{3}$, David R. Kaplan ${ }^{1,7}$ \\ ${ }^{1}$ Program in Neurosciences and Mental Health, The Hospital for Sick Children, Toronto, Canada \\ ${ }^{2}$ Drug Discovery Group, Ontario Institute for Cancer Research, Toronto, ON, Canada \\ ${ }^{3}$ Arnie Charbonneau Cancer Institute, Department of Oncology, Cumming School of Medicine, University of Calgary, Calgary, \\ Alberta, Canada \\ ${ }^{4}$ Canada's Michael Smith Genome Sciences Centre, BC Cancer Agency, Vancouver, Canada \\ ${ }^{5}$ Department of Medical Genetics, University of British Columbia, Vancouver, British Columbia, Canada \\ ${ }^{6}$ Department of Molecular Biology and Biochemistry, Simon Fraser University, Burnaby, British Columbia \\ ${ }^{7}$ Department of Molecular Genetics, University of Toronto, Toronto, ON Canada
}

Correspondence to: David R. Kaplan, email: dkaplan@sickkids.ca

Keywords: glioblastoma, drug discovery, epigenetics, UNC 1999, HDAC inhibitor

Received: March 17, $2016 \quad$ Accepted: July 07, $2016 \quad$ Published: July 18, 2016

\section{ABSTRACT}

Glioblastoma (GBM) is the most lethal and aggressive adult brain tumor, requiring the development of efficacious therapeutics. Towards this goal, we screened five genetically distinct patient-derived brain-tumor initiating cell lines (BTIC) with a unique collection of small molecule epigenetic modulators from the Structural Genomics Consortium (SGC). We identified multiple hits that inhibited the growth of BTICs in vitro, and further evaluated the therapeutic potential of EZH2 and HDAC inhibitors due to the high relevance of these targets for GBM. We found that the novel SAM-competitive EZH2 inhibitor UNC1999 exhibited low micromolar cytotoxicity in vitro on a diverse collection of BTIC lines, synergized with dexamethasone (DEX) and suppressed tumor growth in vivo in combination with DEX. In addition, a unique brain-penetrant class I HDAC inhibitor exhibited cytotoxicity in vitro on a panel of BTIC lines and extended survival in combination with TMZ in an orthotopic BTIC model in vivo. Finally, a combination of EZH2 and HDAC inhibitors demonstrated synergy in vitro by augmenting apoptosis and increasing DNA damage. Our findings identify key epigenetic modulators in GBM that regulate BTIC growth and survival and highlight promising combination therapies.

\section{INTRODUCTION}

Glioblastoma (GBM) is the most lethal and aggressive adult brain tumor. The standard-of-care treatment includes surgical resection, followed by radiotherapy and chemotherapy with the alkylating agent temozolomide (TMZ). Despite this multifaceted therapy, prognosis for GBM patients remains poor with a median survival of only 14.6 months [1]. There are numerous hurdles impeding the development of successful
GBM treatments including high inter- and intra-tumoral heterogeneity $[2,3]$, the inability of $98 \%$ known drugs to cross the blood-brain barrier [4] and the presence of a population of stem cell-like glioma cells, referred to as brain tumor-initiating cells (BTICs), that are thought to contribute to GBM propagation, treatment resistance and tumor recurrence [5-7]. Therefore, to further improve the survival of GBM patients, new therapeutic strategies including combination-based therapies need to be considered. 
Epigenetic mechanisms are being increasingly recognized as a major factor contributing to the pathogenesis of GBM [8,9]. Epigenetic silencing of O-6-methylguanine-DNA methyltransferase (MGMT) by promoter hypermethylation is associated with significantly longer survival [10]. Recent genome-wide genomic and epigenomic analyses have revealed that $46 \%$ of $291 \mathrm{GBM}$ samples tested have at least one somatic mutation in genes associated with chromatin modification [11]. Epigenetic alterations, particularly those involving enzymatic modifications of either DNA or associated histone proteins are currently being exploited for therapeutic drug targeting. However, at the moment there is limited knowledge regarding the mechanisms through which epigenetic modifiers function in GBM, and the possibility of therapeutic targeting has not been rigorously tested for this tumor.

In the current study, we performed a drug screen on five genetically-distinct BTIC lines with a unique collection of small molecule epigenetic inhibitors compiled by the Structural Genomics Consortium (SGC), and validated our findings on these and 10 additional lines. The BTIC lines were generated from tumors from newly diagnosed and recurrent GBM patients [12-17], exhibit the ability to self-renew and differentiate into multiple neural cell lineages [14], encompass the diversity of molecular genetic alterations that are known to occur in human GBM patients (e.g. EGFR, PTEN, p53, IDH1 etc.) and are tumorigenic in orthotopic xenograft murine models $[14,18]$. We identified multiple hits, and further evaluated the therapeutic potential of compounds regulating two epigenetic modifiers, enhancer of zeste homolog 2 (EZH2) and histone deacetylase (HDAC), both of which have high relevance for GBM.EZH2 as well as HDAC1 and HDAC2 are over-expressed in GBM [19$23]$ and are associated with shorter overall survival [19, $20,22]$. We show here that the SAM-competitive EZH2 inhibitor UNC1999 exhibits low micromolar cytotoxicity in vitro on a diverse collection of BTIC lines, synergizes with Dexamethasone (DEX) and suppresses tumor growth in vivo in combination with DEX. Furthermore, we demonstrate that a unique brain-penetrant class I HDAC inhibitor is cytotoxic in vitro on a panel of BTIC lines and is able to extend survival in combination with TMZ in an orthotopic model in vivo. Finally, we demonstrate that the combination of EZH2 and HDAC inhibitors shows synergy in vitro by augmenting apoptosis and increasing DNA damage of GBM tumors.

\section{RESULTS}

\section{Small molecule screen identifies epigenetic modulators that target diverse BTICs}

To identify epigenetic modulators that could inhibit the growth of BTICs in vitro, we screened five genetically distinct patient-derived BTIC lines with an SGC collection of 24 chemical probes (Figure 1A). Compound addition was performed digitally using 12-point serial dilutions, and alamarBlue reduction was performed after 3 or 6 days to assess metabolic activity as an indicator of cell health. Compounds exhibiting $\mathrm{IC}_{50}$ values lower than $5 \mu \mathrm{M}$ in either the 3 day or a 6-day screening assays were defined as hits (marked in red, Figure 1B). Compounds targeting multiple epigenetic targets were identified that suppressed BTIC health at low micromolar or nanomolar ranges, including histone methyl transferases (G9A, EZH2, SMYD2), BET bromodomains, histone demethylase (JMJD3) and histone deacetylases (HDACs). Compounds that targeted BET ((+)-JQ1) and JMJD3 (GSKJ4) have been recently described as promising compounds for GBM and pediatric GBM [24-26], and therefore we decided to not pursue them further. We tested the compounds targeting G9A and SMYD2 for their putative target inhibition (H3K9me2 and H3K4me1/H3K36me2, respectively) and found them inactive, suggesting that their cytotoxicity could be due to off-target effects. Therefore, we decided to focus on novel compounds targeting EZH2 and HDAC, both of which have high relevance for GBM [19-22, 27].

\section{Targeting EZH2 in BTICs using a novel SAM- competitive inhibitor UNC1999}

EZH2 is the catalytic subunit of the Polycomb Repressive Complex 2 (PRC2) that inhibits gene expression through tri-methylation of lysine 27 on histone H3 (H3K27me3). Deregulation of EZH2 has been documented in various cancer types, both solid and hematological, and is associated with poor survival [28-30]. Therefore, we first assessed EZH2 expression in a panel of genetically-diverse BTIC lines. We observed EZH2 mRNA (Figure 2A) and protein (Figure 2B) in all BTIC lines tested, with virtually no expression in skin-derived precursor cells (SKPs), which are primary postnatal human neural-like dermal stem cells used as a normal neural stem cell control. While we did not observe in BTIC lines EZH2 activating point mutations Y641 and A677 that occur in hematological malignancies, an increase in copy number variations (CNV) was detected in the majority of those lines (Supplementary Table 1).

We next compared the SAM-competitive EZH2 inhibitors GSK126, GSK343, UNC1999 and EPZ6438 on 10 BTIC lines for their ability to suppress cell health in the alamarBlue assay. UNC1999, a SAM-competitive EZH2/EZH1 inhibitor [31] identified in our screening of the SGC library, exhibited superior ability to suppress cell health as compared to the other inhibitors $\left(\mathrm{IC}_{50}=2-5\right.$ $\mu \mathrm{M}$; Figure 2C), with EPZ6438 being inactive even at 10 $\mu \mathrm{M}$ (data not shown). UNC2400, an inactive analogue of UNC1999 [29, 31], did not affect cell growth at $10 \mu \mathrm{M}$ or lower, suggesting that the biological effects of UNC1999 
were specific to its activity as a H3K27 trimethylation inhibitor. To address why other EZH2 inhibitors did not show similar efficacy to UNC1999, we compared the target inhibition of UNC1999 with three other EZH2 inhibitors, GSK343, GSK126 and EPZ-6438 at the same concentrations $(2 \mu \mathrm{M}$ and $3 \mu \mathrm{M})$ on BTICs. We assessed different potential targets including $\mathrm{H} 3 \mathrm{~K} 27 \mathrm{me} 3$, $\mathrm{H} 3 \mathrm{~K} 27 \mathrm{me} 2$, H3K9Me2, H3K4Me1 and H3K27ac as well as EZH2 and total histone H3 (Supplementary
Figure 1). UNC1999 inhibited only trimethylation of H3K27 without affecting the other targets or altering the total level of EZH2 or Histone H3. The other three inhibitors suppressed trimethylation of H3K27, GSK126 and EPZ-6438 also inhibited dimethylation of H3K27, and GSK343 also reduced H3K9me2 and H3K4me1 levels. Our conclusion is that UNC1999 is more specific than the other inhibitors and therefore, we focused on UNC1999 for our further studies.

A.

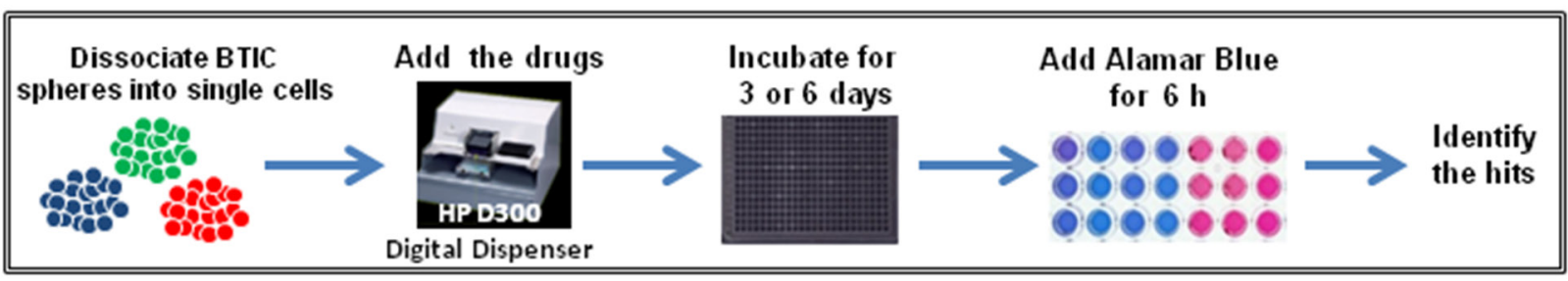

B.

\begin{tabular}{|c|c|c|c|c|c|c|c|c|c|c|c|c|}
\hline \multirow{3}{*}{ Number } & \multirow{3}{*}{ Compound name } & \multirow{3}{*}{ Putative target } & \multicolumn{10}{|c|}{$1 \mathrm{C50}(\mathrm{pM})$} \\
\hline & & & \multicolumn{2}{|c|}{ BT143 } & \multicolumn{2}{|c|}{ BT53 } & \multicolumn{2}{|c|}{ BT69 } & \multicolumn{2}{|c|}{ BT100 } & \multicolumn{2}{|c|}{ BT194 } \\
\hline & & & $3 d$ & $6 d$ & $3 d$ & $6 d$ & $3 d$ & $6 d$ & $3 d$ & $6 d$ & $3 d$ & 6d \\
\hline 1 & UNC0638 & G9a & 1.2 & 0.7 & 2.1 & 1.2 & 2 & 0.6 & 2.1 & 1.2 & 2.6 & 1.6 \\
\hline 2 & UNC0642 & G9a & 2.9 & 1.2 & 4.9 & 1.4 & 3.6 & 1.2 & 4.7 & 1.9 & 7.9 & 2.9 \\
\hline 3 & A366 & G9a & $>10$ & $>10$ & $>10$ & $>10$ & $>10$ & $>10$ & $>10$ & $>10$ & $>10$ & $>10$ \\
\hline 4 & SGC946 & Dot1L & 9.7 & 5.1 & 10 & 10 & 10 & 4.9 & 10 & 5.8 & 10 & 10 \\
\hline 5 & UNC1999 & $\mathrm{EZH} 2$ & 2.6 & 2.4 & 4.7 & 2.4 & 2.7 & 1.9 & 3.2 & 2.3 & 5 & 3.6 \\
\hline 6 & GSK343 & EZH2 & 4 & 3.2 & 10 & 5.6 & 4.9 & 2.6 & 4.9 & 4.4 & 10 & 6.2 \\
\hline 7 & JQ1 & BET & $>10$ & 0.1 & $>10$ & 0.1 & $>10$ & 0.7 & $>10$ & 0.06 & $>10$ & $>10$ \\
\hline 8 & PFI-1 & BET & $>10$ & 1.5 & $>10$ & 3.7 & $>10$ & 10 & $>10$ & 1.7 & $>10$ & $>10$ \\
\hline 9 & GSK2801 & BAZ2A/B & $>10$ & $>10$ & $>10$ & $>10$ & $>10$ & $>10$ & $>10$ & $>10$ & $>10$ & $>10$ \\
\hline 10 & Dacinostat & HDACs & 0.04 & 0.01 & 0.02 & 0.01 & 0.02 & 0.01 & 0.03 & 0.01 & 0.06 & 0.03 \\
\hline 11 & GSK J4 & JMJD3 & 1.3 & 1.1 & 1.2 & 1.7 & 1.6 & 1.3 & 1.9 & 1.4 & 1.2 & 1.2 \\
\hline 12 & $10 \times 1$ & Demethylases 20G & $>10$ & $>10$ & $>10$ & $>10$ & $>10$ & $>10$ & $>10$ & $>10$ & $>10$ & $>10$ \\
\hline 13 & $10 \times 2$ & PHD2 & $>10$ & $>10$ & $>10$ & $>10$ & $>10$ & $>10$ & $>10$ & $>10$ & $>10$ & $>10$ \\
\hline 14 & PFI-2 & SETD7 & $>10$ & $>10$ & $>10$ & $>10$ & $>10$ & $>10$ & $>10$ & $>10$ & $>10$ & $>10$ \\
\hline 15 & ICBP112 & CBP/EP300 bromo & $>10$ & $>10$ & $>10$ & $>10$ & $>10$ & $>10$ & $>10$ & $>10$ & $>10$ & $>10$ \\
\hline 16 & C646 & p300/CBP & $>10$ & 7.9 & $>10$ & 7.4 & $>10$ & 5.2 & $>10$ & 4.1 & 6.7 & 4.4 \\
\hline 17 & PFI-3 & Smarca2 $/ 4$ & $>10$ & $>10$ & $>10$ & $>10$ & $>10$ & $>10$ & $>10$ & $>10$ & $>10$ & $>10$ \\
\hline 18 & LLY-507 & SMYD2 & 2.3 & 1.2 & 2.2 & 1.6 & 1.3 & 1.3 & 1.6 & 1.1 & 1.7 & 1.5 \\
\hline 19 & OICR-9429 & WDR5 & $>10$ & $>10$ & $>10$ & $>10$ & $>10$ & $>10$ & $>10$ & $>10$ & $>10$ & $>10$ \\
\hline 20 & SGC 707 & PRMT3 & $>10$ & 4.6 & $>10$ & 10 & $>10$ & 2.0 & $>10$ & $>10$ & $>10$ & $>10$ \\
\hline 21 & Olaparib & PARP1/2 & $>10$ & $>10$ & $>10$ & 10 & 2.8 & 0.1 & $>10$ & 0.9 & $>10$ & 0.4 \\
\hline 22 & OF-1 & BRPF1-3 & $>10$ & $>10$ & 10 & 1.9 & 10 & 10 & $>10$ & $>10$ & $>10$ & $>10$ \\
\hline 23 & $\mathrm{Cl} 994$ & HDAC1 & 10 & 2.4 & 10 & 2.5 & 2.5 & 1.7 & $>10$ & 2.6 & $>10$ & $>10$ \\
\hline 24 & Bromosporine & BRD 2, 4, 9 & $>10$ & 1.1 & 2.5 & 1.3 & 3.5 & 1.2 & 10 & 1.1 & $>10$ & 2.1 \\
\hline
\end{tabular}

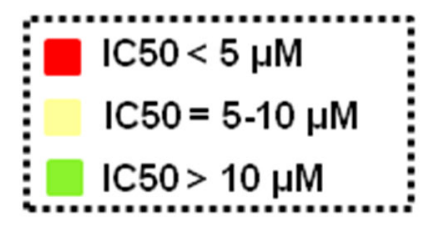

Figure 1: Testing of the SGC epigenetic library of chemical probes on five BTICs lines. A. Detailed design of the screening procedure; B. Epigenetic screen results. Compounds that exhibit $\mathrm{IC}_{50}<5 \mu \mathrm{M}$ were defined as hits and are shown in red, drugs with $5 \mu \mathrm{M}<$ $\mathrm{IC}_{50}<10 \mu \mathrm{M}$ are marked in yellow and inactive compounds that exhibit $\mathrm{IC}_{50}>10 \mu \mathrm{M}$ are shown in green. 


\section{UNC1999 treatment of BTICs decreases cell} viability, impairs self-renewal, causes cell cycle arrest and decreases $\mathrm{H} 3 \mathrm{~K} 27 \mathrm{Me} 3$ levels

We first asked whether treatment of BTICs with UNC1999 affected cell viability. The BT73 line was treated with increasing concentrations of UNC1999 (4-6 $\mu \mathrm{M})$ and viable cells were counted at 72 and 96 hours post-treatment. UNC1999 significantly decreased viable cell numbers in a dose-dependent manner as compared to DMSO-treated cells at all time points tested (Figure 3A). Next, the effect of UNC1999 on self-renewal was investigated. For this, BT73 and BT147 cells were treated with varying doses of the inhibitor and the number of spheres counted after 6 days. UNC1999 completely abrogated sphere formation at $5 \mu \mathrm{M}$ in both BTIC lines (Figure 3B). To ask whether UNC1999 also had cytostatic effects on BTIC lines, the percentage of cells in the different stages of the cell cycle was examined by flow cytometry. BT73 cells were treated with varying concentrations of UNC1999 (2-5 $\mu \mathrm{M})$, and cellular DNA content was examined 48 hours later. The percentage of cells in the $G_{1}$ phase of the cell cycle significantly increased as a consequence of drug treatment, with a slight decrease in the percentage of cells in both $\mathrm{S}$ and $\mathrm{G}_{2} / \mathrm{M}$ phases (Figure $3 \mathrm{C}$ ). In contrast, there were no changes in the percentage of cells at different stages of the cell cycle in cells treated with UNC2400, an inactive analogue of UNC1999 [29, 31]. In BTICs, UNC1999 treatment completely inhibited trimethylation of lysine 27 on Histone H3 (H3K27) without affecting the total levels of either Histone H3 or EZH2 (Figure 3D). Although there were no changes detected in the expression of the apoptotic marker cleaved-PARP as a consequence of drug treatment, we observed an increase in the expression of LC3B II, suggesting that the potential mechanism of cell death could be autophagy rather than apoptosis (Figure 3D), in agreement with the reported autophagic
A.

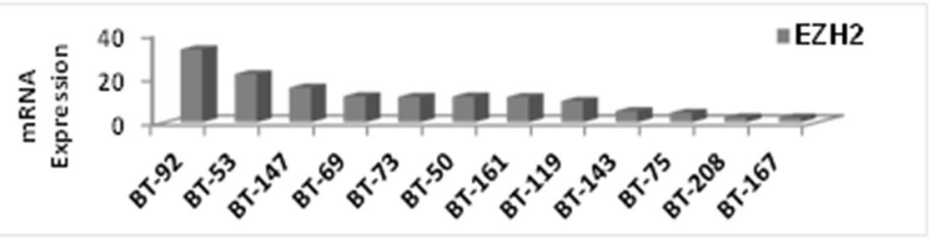

B.

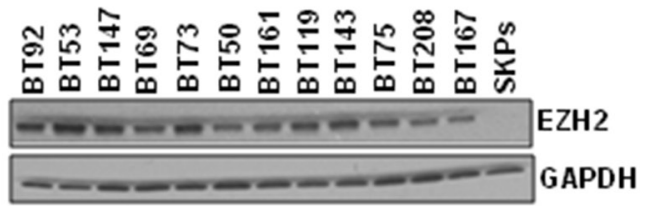

C.

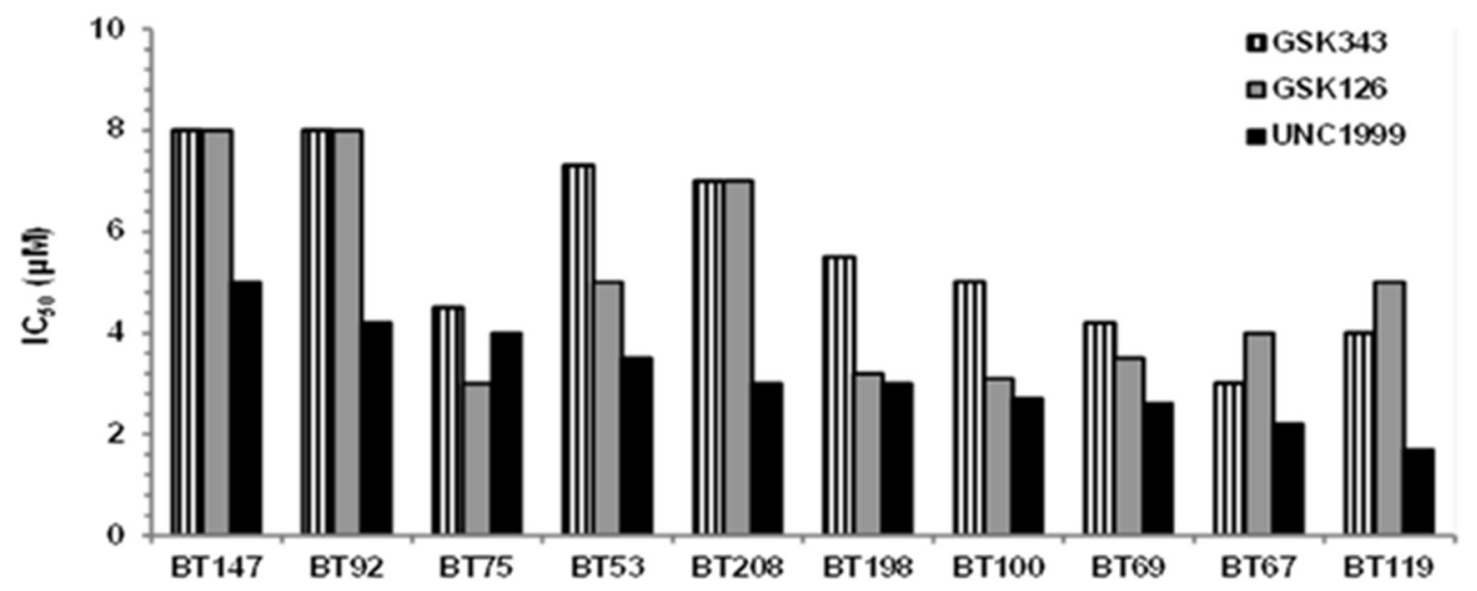

UNC1999

GSK126

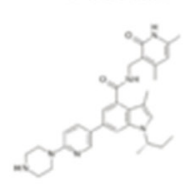

GSK343

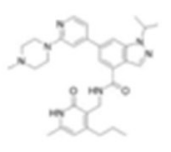

Figure 2: EZH2 is overexpressed in BTICs and SAM-competitive EZH2 inhibitors, in particular UNC1999, are cytotoxic in vitro. A. EZH2 mRNA expression in BTICs. B. EZH2 protein expression in BTICs and SKPs by immunoblotting with antiEZH2 antibody. C. $\mathrm{IC}_{50}$ values presented as bar graph for SAM-competitive EZH2 inhibitors using alamarBlue as a read-out. 
A.

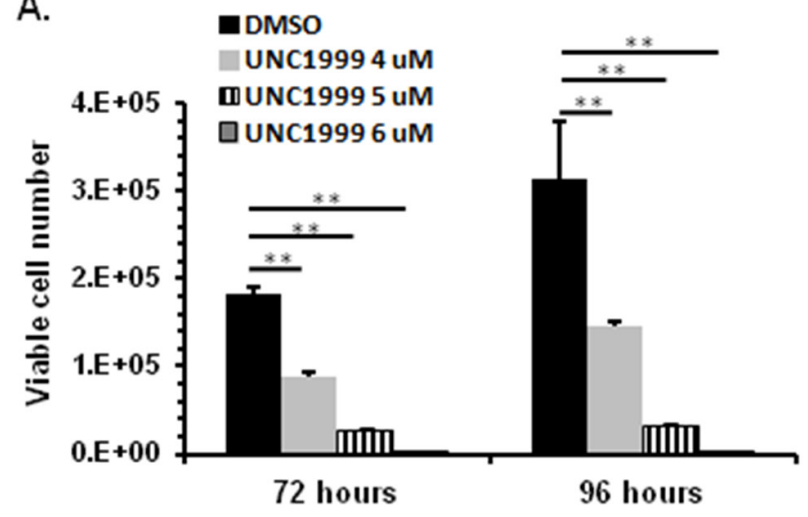

C.

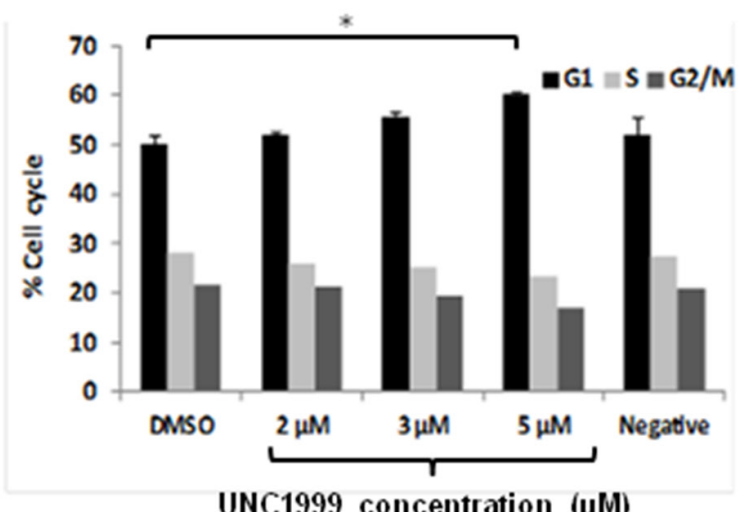

UNC1999 concentration ( $\mu$ M)

E.

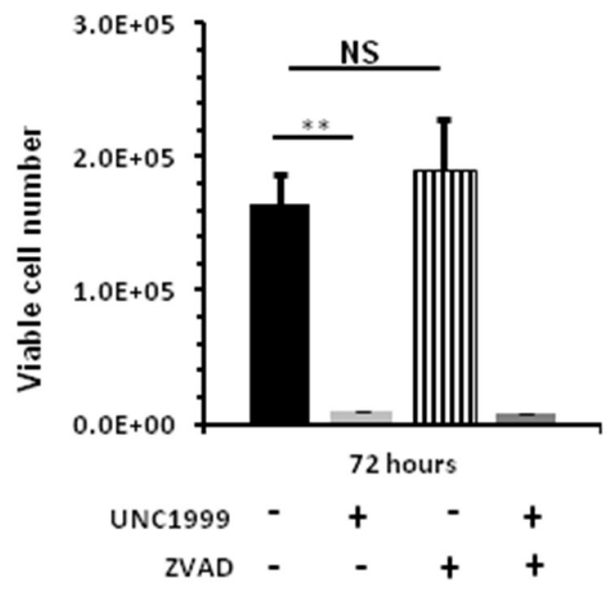

B.

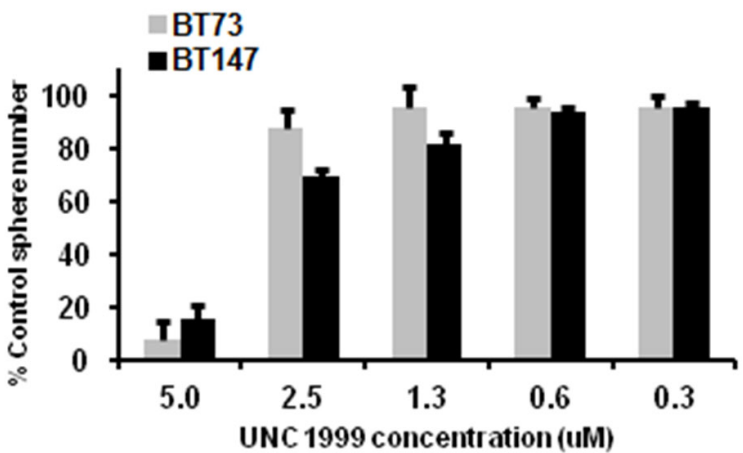

D.

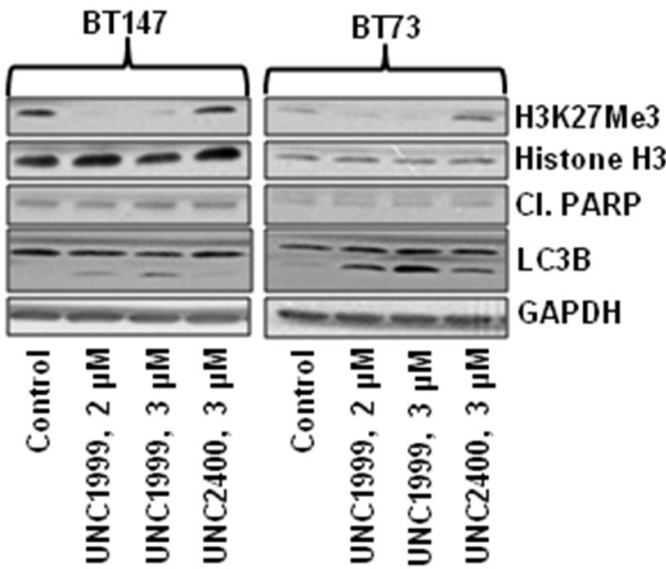

F.

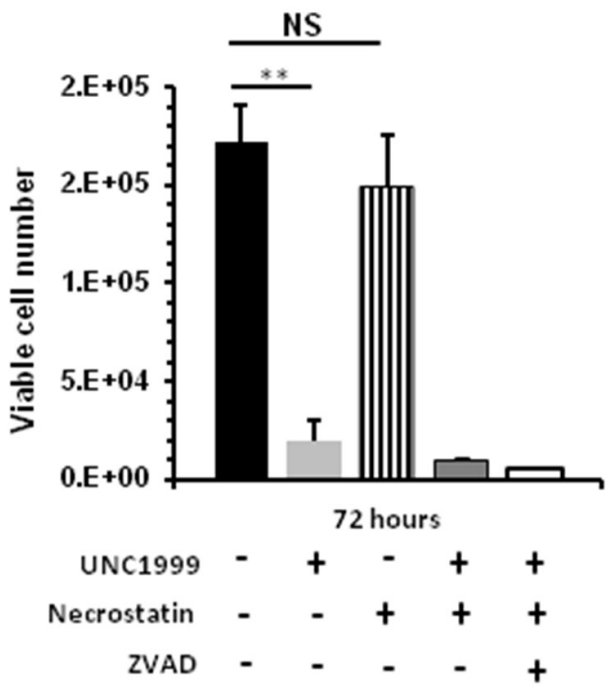

Figure 3: UNC1999 treatment decreases cell viability, impairs self-renewal, causes cell cycle arrest and decreases H3K27Me3. A. Treatment with UNC1999 $(4-6 \mu \mathrm{M})$ reduces viable cell numbers in BT73 as assessed by trypan blue exclusion $(\mathrm{n}=3$; ** $\mathrm{P}<0.01)$. B. Treatment with varying concentrations of UNC1999 impairs self renewal in BT73 and BT147 as assessed by sphere assay $(\mathrm{n}=3)$. C. Treatment with UNC1999 $(2-5 \mu \mathrm{M})$ induces G1 cell cycle arrest in BT73 as assessed by propidium iodide staining. D. Representative western blot demonstrates the effect of treatment with UNC1999 and the negative control UNC2400 on H3K27Me3, total levels of Histone H3, cleaved-PARP and LC3B in BT73 and BT147. E. Treatment with UNC1999 (5 $\mu$ M) and ZVAD-FMK (20 $\mu$ M) for 72 hours does not rescue cell viability in BT73 as assessed by trypan blue exclusion ( $\mathrm{n}=3 ; * * \mathrm{P}<0.01)$. F. Treatment with $\mathrm{UNC1} 1999(5 \mu \mathrm{M})$ and Necrostatin $(50 \mu \mathrm{M})$ for 72 hours does not rescue cell viability in BT73 as assessed by trypan blue exclusion $(\mathrm{n}=3 ; * * \mathrm{P}<0.01)$. 
mechanism of GSK343 and UNC1999-mediated cell death in breast and lung carcinoma cell lines [32]. In order to rule out apoptosis and necroptosis, we used Annexin V assay to assess the percentage of apoptotic cells. We could not detect Annexin V-positive cells at either 24, 48 or 72 hours following UNC1999 treatment (data not shown). Moreover, co-incubation of cells with UNC1999 and pancaspase inhibitor Z-VAD-FMK did not rescue the cells from dying (Figure 3E), suggesting that UNC1999 induces cell death independently of apoptosis. Necroptosis was apparently not involved in UNC1999-induced cell death. Co-incubation of cells with Necrostatin, a necroptosis inhibitor [33], failed to rescue UNC1999-induced cell death (Figure 3F), and co-incubating the cells with UNC1999 and both Necrostatin and Z-VAD-FMK also did not have an effect on cell viability, confirming that these mechanisms are not compensating for each other. Since UNC1999 increased the expression of the autophagic marker LC3B II, we suggest that autophagy rather than necroptosis or apoptosis is the mechanism of BTIC cell death by this compound.

\section{The combination of UNC1999 and dexamethasone is synergistic in vitro and suppresses tumor growth in vivo}

We next asked whether UNC1999 efficacy in vitro can be augmented through combination with additional drugs. We first tested a combination of UNC1999 and Temozolomide (TMZ), a known GBM chemotherapeutic agent, on two BTIC lines (BT73 and BT147). The results were analyzed using the CalcuSyn median effect model, where the $\mathrm{CI}$ indicates synergysm $(\mathrm{CI}<0.9)$, additivity $(\mathrm{CI}=0.9-1.1)$ and antagonism $(\mathrm{CI}>1.1)$. We found that at all concentrations tested there was no synergy or additivity detected in a combination of UNC1999 and TMZ with $\mathrm{CI}$ values at the $\mathrm{ED}_{50}=2.09, \mathrm{ED}_{75}=1.38$ and $\mathrm{ED}_{90}=1.19$ in BT73 and $\mathrm{ED}_{50}=1.2, \mathrm{ED}_{75}=1.19$ and $\mathrm{ED}_{90}=1.19$ in BT147 (Supplementary Figure 2). We then examined a combination of UNC1999 with Dexamethasone (DEX), a corticosteroid commonly used to treat brain edema in GBM patients. It has been previously shown that a combination of a different EZH2 inhibitor, EPZ-6438, was synergistic with glucocorticoid receptor agonists such as prednisolone and dexamethasone in B cell lymphoma [34]. In agreement with those findings, we found that a combination of UNC1999 with DEX was synergistic in two different BTIC lines in vitro with $\mathrm{CI}$ values at the $\mathrm{ED}_{50}=0.87, \mathrm{ED}_{75}=0.82$ and $\mathrm{ED}_{90}=0.78$ in $\mathrm{BT73}$ and $\mathrm{ED}_{50}=0.84, \mathrm{ED}_{75}=0.78$ and $\mathrm{ED}_{90}=0.73$ in BT147 (Figure 4A). There were no additional effects on $\mathrm{H} 3 \mathrm{~K} 27 \mathrm{me} 3$ levels as a consequence of combination (Figure 4B), nor were there changes in EZH2 protein levels, total Histone $\mathrm{H} 3$ or cleaved-PARP. We did observe a decrease in c-MYC protein expression following treatment with the combination of drugs, although DEX alone was also able to suppress c-MYC.
Moreover, we observed no additional increase in LC3B II. To investigate further potential autophagy mechanisms, the effect of UNC199 or DEX alone and in combination on p62/SQTM1, a known autophagy substrate that decreases as a consequence of ongoing autophagy, was examined. We found that in both BT73 and BT147 lines, p62 levels increased in the combination group as compared to the DMSO control and with the single drugs alone (Supplementary Figure 3). These findings are similar to other reports showing that impairment of autophagic flux results in autophagy-induced cell death $[35,36]$.

We next evaluated the efficacy of UNC1999 treatment alone and in combination with DEX in vivo. To assess whether UNC1999 crossed the blood brain barrier and accumulated to sufficient concentrations in vivo, we performed a pharmacokinetic analysis of plasma and brain samples using LC/MS. Following oral administration of $150 \mathrm{mg} / \mathrm{kg}$, a dose in which UNC1999 elicited no toxicity, the concentration in the plasma was $\sim 7 \mu \mathrm{M}$ and only 0.5 $\mu \mathrm{M}$ in the brain at both $30 \mathrm{~min}$ and 4 hours. Since this brain concentration was 10 -fold less than the in vitro $\mathrm{IC}_{50}$ on the BTIC lines, we did not proceed with an orthotopic model. We instead tested its efficacy in a flank xenograft model where we found that the concentration of the drug in the tumor was $\sim 13 \mu \mathrm{M}$, as a proof of concept. For this analysis, NOD/SCID mice with small established BT73 tumors were treated with either vehicle, UNC1999 alone $(150 \mathrm{mg} / \mathrm{kg})$, DEX alone $(1 \mathrm{mg} / \mathrm{kg})$ or a combination of the two drugs for 17 days. To evaluate target inhibition in vivo, we isolated tumors 10 days after initiation of treatment with $150 \mathrm{mg} / \mathrm{kg}$ of UNC1999. UNC1999 decreased the trimethylation of $\mathrm{H} 3 \mathrm{~K} 27$ in a similar fashion to that observed in vitro (Supplementary Figure 4), indicating that UNC1999 exhibits potent target inhibition both in vitro and in vivo. As shown in Figure 4C, UNC1999 alone was ineffective at suppressing tumor growth. Treatment with DEX alone had only a partial effect, while treatment with both UNC1999 and DEX significantly suppressed tumor growth as compared to control or single-agent treatment.

\section{HDAC inhibitor (compound 26) treatment of BTICs decreases cell viability, impairs self-renewal, causes cell cycle arrest, induces apoptosis and increases acetylation of histone $\mathrm{H3}$}

Two class I HDAC inhibitors were identified as hits from the epigenetic screen, Dacinostat and CI994. Both HDAC1 and HDAC2 mRNA and protein were detected in most of the BTIC lines tested, and were absent in pediatric skin-derived precursor cells (SKPs) (Supplementary Figure 5). Sequencing of BTIC lines revealed that the BT147 line encoded a point mutation in HDAC2, and several lines had an increase in HDAC2 copy number variations (CNV) (Supplementary Table 1). Since blood-brain-barrier (BBB) penetration is one of the obstacles impeding successful implementation of HDAC inhibitors in the clinic, we tested 


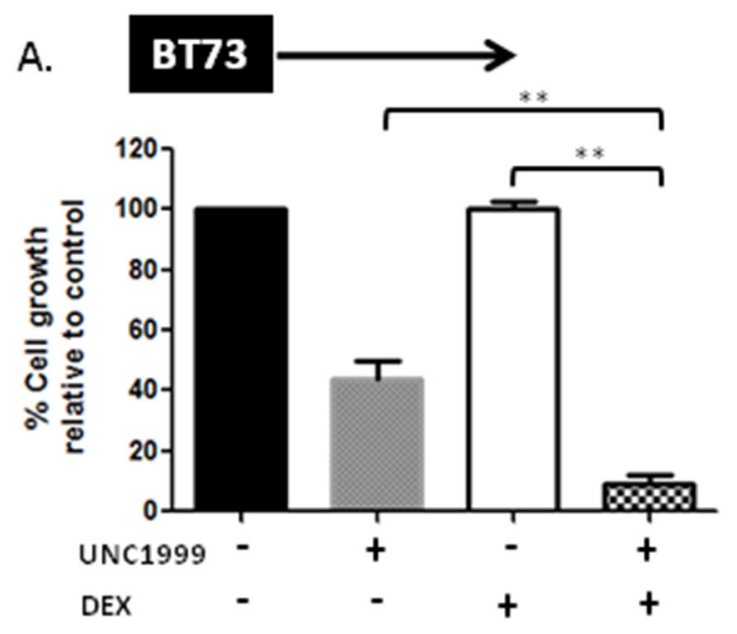

\section{BT147}

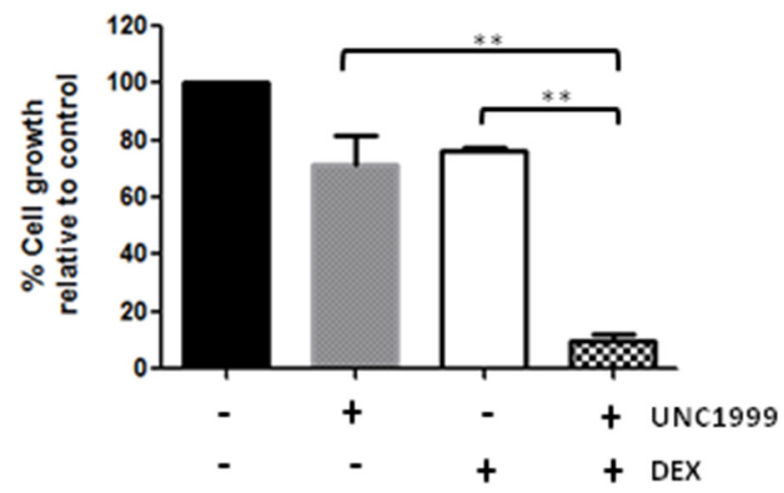

B.
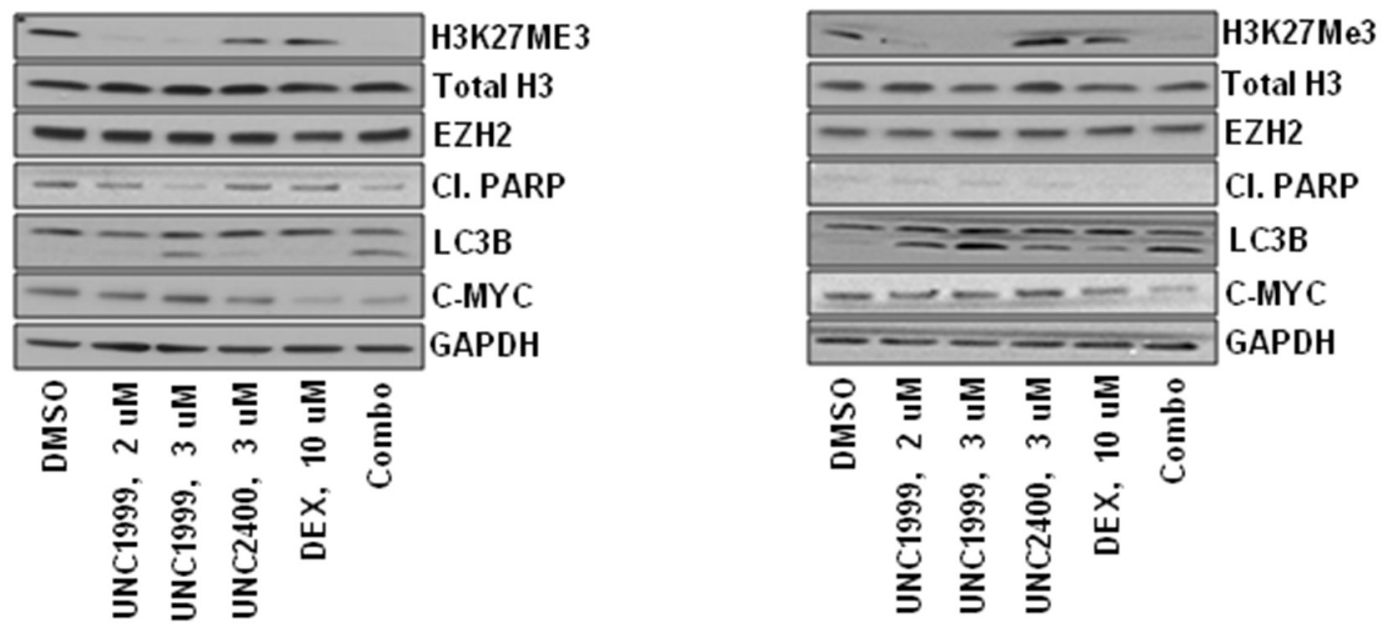

C.
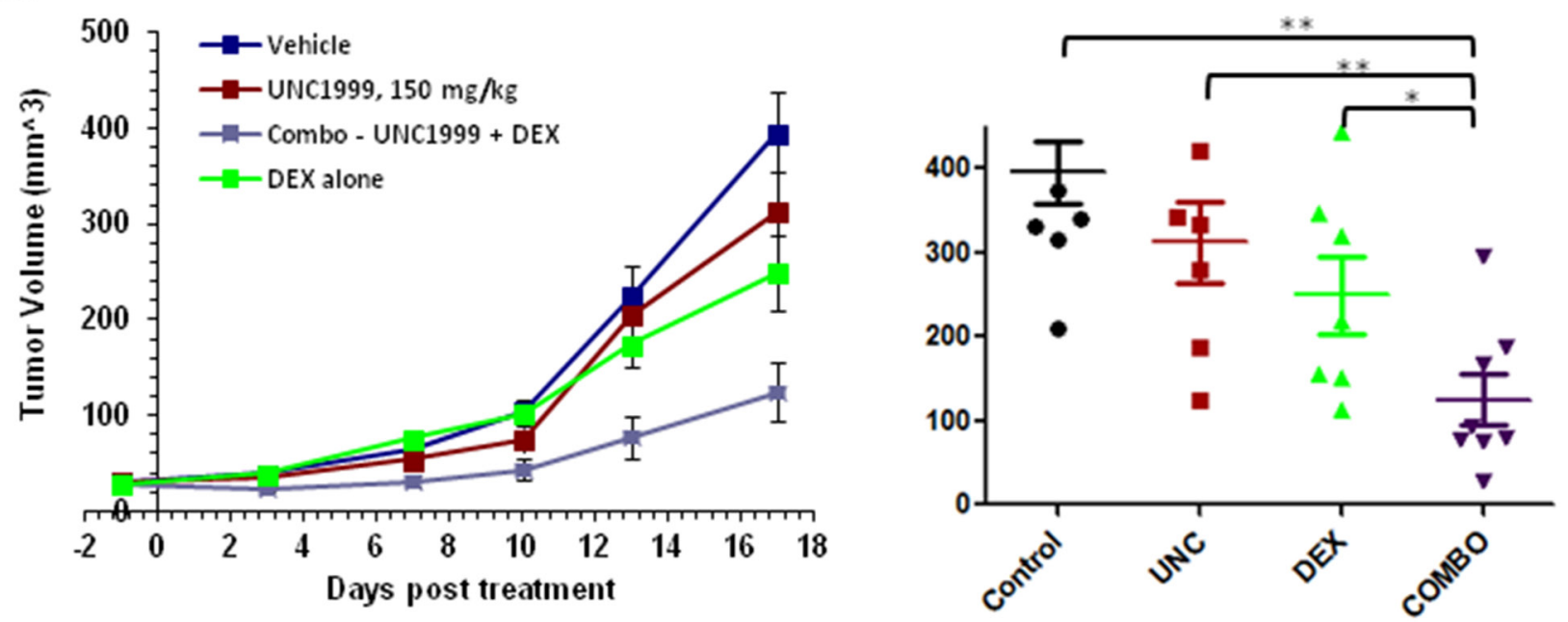

Figure 4: UNC1999 is synergistic with dexamethasone (DEX) in vitro and suppresses tumor growth in vivo in a flank xenograft model. A. Representative bar graphs demonstrating synergy between UNC1999, $3.7 \mu \mathrm{M}$ and DEX, $31 \mu \mathrm{M}$ in vitro. B. Representative western blots demonstrating assessment of different targets following treatment with drugs alone and a combination in vitro. C. NOD/SCID mice bearing $25 \mathrm{~mm}^{3}$ tumors were randomized into 4 groups: 1) vehicle; 2) UNC1999, $150 \mathrm{mg} / \mathrm{kg}$, gavage; 3) DEX, $1 \mathrm{mg} / \mathrm{kg}$, i.p. and 4) combination. Three independent experiments were performed. Representative tumor growth data and tumor volume at 2 weeks after treatment initiation are shown $\left({ }^{*} \mathrm{p}<0.05 ; * * \mathrm{p}<0.01\right)$. 


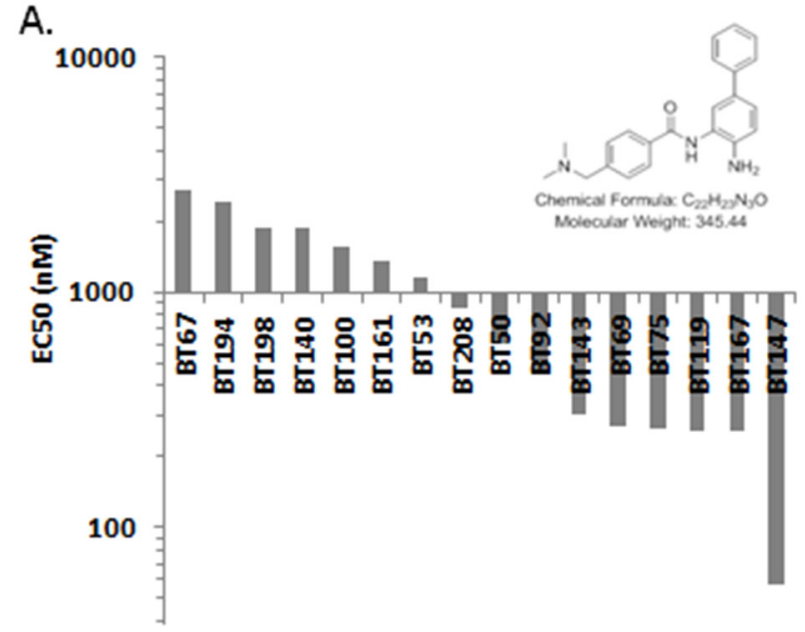

C.

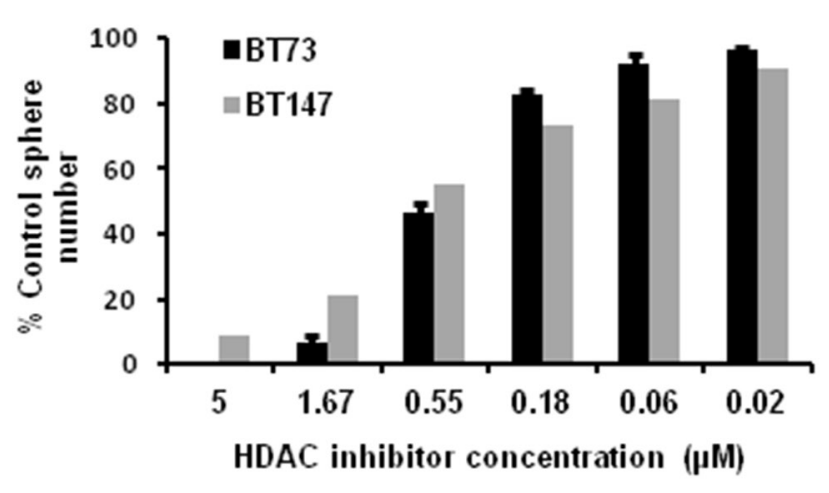

E.

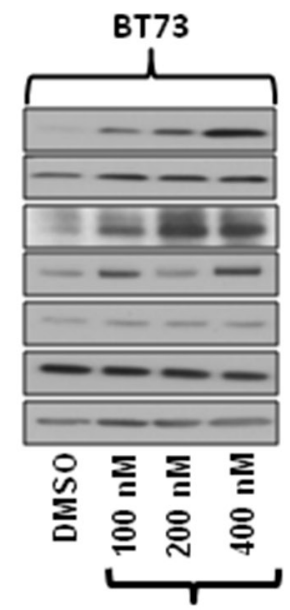

HDAC inhibitor
B.

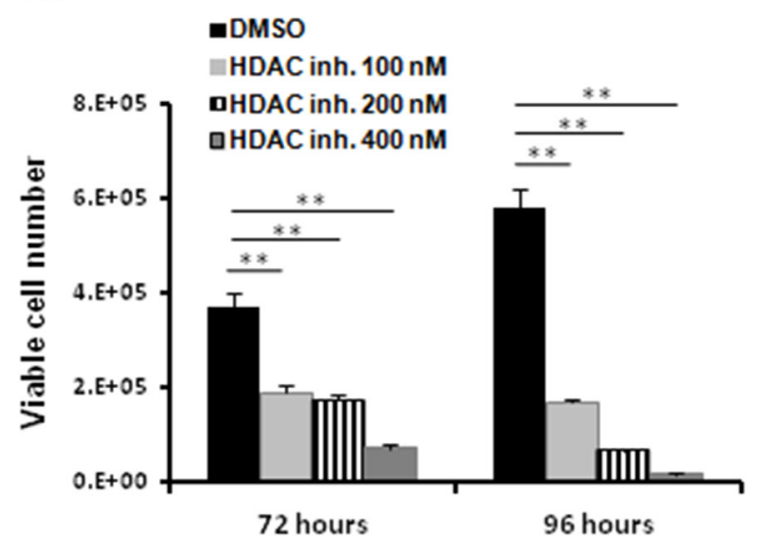

D. $\square \mathrm{G} \backsim \mathrm{S} \backsim \mathrm{G} 2 / \mathrm{M}$

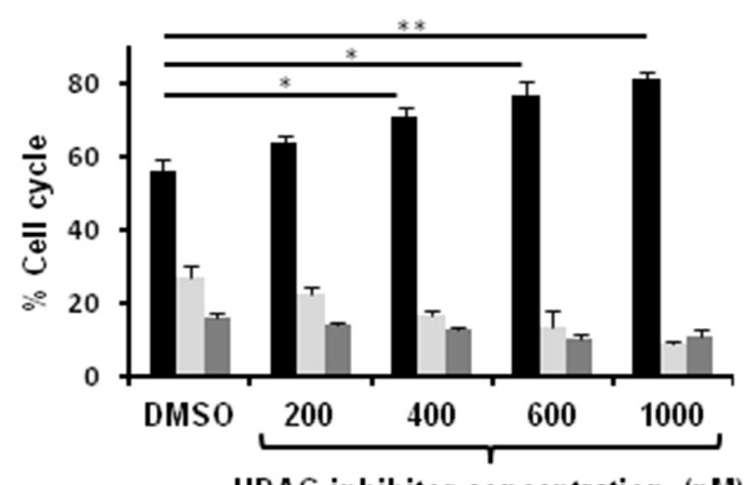

HDAC inhibitor concentration (nM)

F.

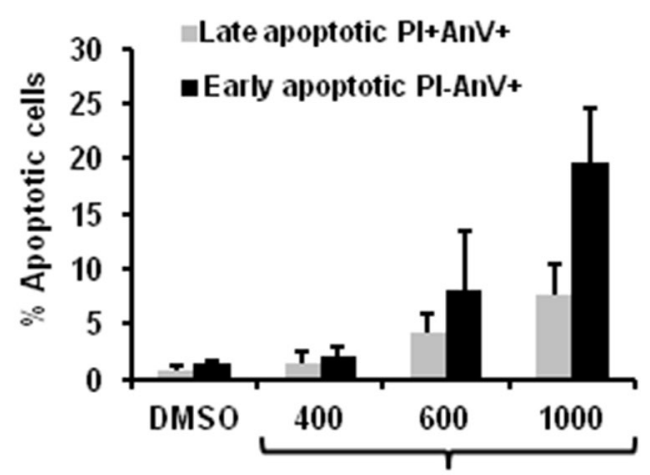

HDAC inhibitor concentration (nM)

Figure 5: Treatment with brain-penetrant HDAC inhibitor compound 26 decreases cell viability, impairs self-renewal, causes cell cycle arrest, induces apoptosis and increases total levels of acetylated H3. A. Compound 26 is cytotoxic on diverse BTIC lines in vitro. Waterfall graph with $\mathrm{IC}_{50}$ values is shown. B. Treatment with compound $26(100-400 \mathrm{nM})$ reduces viable cell numbers in BT73 as assessed by trypan blue exclusion $\left(\mathrm{n}=3 ;{ }^{* *} \mathrm{P}<0.01\right)$. C. Treatment with varying concentrations of compound 26 impairs self renewal in BT73 and BT147 as assessed by sphere assay $(n=3)$. D. Treatment with compound 26 (200-1000 nM) induces G1 cell cycle arrest in BT73 as assessed via Propidium iodide staining. E. Representative western blot demonstrates effect of treatment with compound 26 (100$400 \mathrm{nM}$ ) on total acetylation of histone $\mathrm{H} 3$, total levels of Histone H3, HDAC1/2, p21 and cleaved PARP in BT73 and BT147. F. Treatment with compound $26(400-1000 \mathrm{nM})$ increases the percentage of early and late apoptotic cells as assessed via Annexin $\mathrm{V}$ staining $(\mathrm{n}=3)$. 
an analogue of Entinostat, a known clinical HDAC inhibitor, optimized to improve BBB penetration in a baboon model [37]. This compound (referred to herein as "compound 26") has been reported to inhibit recombinant human HDAC1 and HDAC2 at nanomolar concentrations [37]. Compound 26 suppressed metabolic activity as determined by alamarBlue assay in a panel of 15 BTIC lines with $\mathrm{IC}_{50}$ values ranging from $57 \mathrm{nM}$ to $2735 \mathrm{nM}$ (Figure 5A). BT147 was identified as the most sensitive line, possibly due to the presence of a point mutation in HDAC2 which was also detected in the original patient tumor. Therefore, we focused our studies on the brain-penetrant HDAC inhibitor compound 26.

We first asked whether treatment with compound 26 affected cell viability. BT73 cells were treated with varying concentrations of compound 26 (100-400 nM) and cell viability was determined at 72 and 96 hours post-treatment. The inhibitor significantly decreased cell viability in a dose and time-dependent manner (Figure 5B). To determine whether compound 26 also affected self-renewal, BT73 and BT147 cells were treated with different doses of the inhibitor, and the number of spheres was quantified after 6 days. Compound 26 completely abrogated sphere formation at $5 \mu \mathrm{M}$ in both BTIC lines (Figure 5C). We next examined the percentage of cells found at the different stages of the cell cycle. BT73 cells were treated with varying concentrations of compound 26 (200 nM-1000 nM) and cellular DNA content examined 72 hours later. The percentage of cells in the $\mathrm{G}_{1}$ phase of the cell cycle significantly increased while the percentage in both $\mathrm{S}$ and $\mathrm{G}_{2} / \mathrm{M}$ phases was decreased (Figure 5D). Treatment with compound 26 induced hyperacetylation of Histone $\mathrm{H} 3$ in a dose-dependent manner, without affecting the total levels of Histone H3, HDAC1 or HDAC2 (Figure 5E). We also observed an increase in the levels of the cyclin-dependent kinase inhibitor $\mathrm{p} 21^{\mathrm{CIP} 1 / \mathrm{WAF} 1}$ and cleaved-PARP, suggesting cell cycle arrest and apoptosis as potential mechanisms of action of the inhibitor (Figure $5 \mathrm{E})$. In order to further confirm the mechanism of cell death, we used Annexin $\mathrm{V}$ assay to assess the percentage of apoptotic cells (Figure 5F). BT73 cells were treated with varying concentrations of compound 26 (400 nM-1000 $\mathrm{nM}$ ) and the percentage of both early (Annexin V-positive, PI-negative) and late (Annexin V-positive, PI-positive) apoptotic cells was quantified 72 hours later. The percentage of early and late apoptotic cells significantly increased in a dose-dependent manner as compared to vehicle-treated cells, confirming that compound 26 induced cell death through apoptosis.

\section{The HDAC inhibitor compound 26 crosses the BBB, accumulates in the brain and induces a significant survival benefit in vivo in combination with TMZ}

Since compound 26 is known to cross the BBB in baboons, we determined the concentration that accumulates in mouse brain and the dose suitable for in vivo efficacy studies. A range of doses (3, 10, 20 and $40 \mathrm{mg} / \mathrm{kg}$ ) was administered in vivo over 5 days by oral gavage. Plasma and brains were collected and analyzed by LC/MS. Micromolar concentrations of the drug were detected in both the plasma and the brain (Figure 6A). We chose $10 \mathrm{mg} / \mathrm{kg}$ for in vivo studies, since the brain concentration achieved at this dose level at 3 hours was 10 -fold over the in vitro $\mathrm{IC}_{50}$ of compound 26 efficacy of seven BTIC lines. To determine in vivo efficacy, NOD/ SCID mice with small established BT147 intracranial tumors were randomized into 4 groups and treated with 1) vehicle, 2) compound 26 alone (10 mg/kg), 3) TMZ alone $(50 \mathrm{mg} / \mathrm{kg})$ or 4) combination of the two drugs for a total of 3 cycles ( 5 days ON, 2 days OFF). As shown in Figure $6 \mathrm{~B}$, treatment with compound 26 alone was ineffective in extending survival. Treatment with TMZ alone had a partial effect, while treatment with both compound 26 and TMZ was able to significantly extend survival as compared to control (approximately 20 days) or singleagent treatment (by approximately 10 days).

\section{HDAC inhibitor compound 26 is synergistic with UNC1999 in vitro}

Lastly, we examined the efficacy of combining UNC1999 and the HDAC inhibitor. We hypothesized that inhibition of two distinct epigenetic pathways might complement each other and result in strong synergism. Indeed, a combination of UNC1999 with the HDAC inhibitor demonstrated strong synergism in two different $\mathrm{BTIC}$ lines in vitro with $\mathrm{CI}$ values at the $\mathrm{ED}_{50}=0.55$, $\mathrm{ED}_{75}=0.49$ and $\mathrm{ED}_{90}=0.48$ in $\mathrm{BT73}$ and $\mathrm{ED}_{50}=0.35$, $\mathrm{ED}_{75}=0.30$ and $\mathrm{ED}_{90}=0.27$ in $\mathrm{BT} 147$ (Figure 7A). Similarly, we observed either synergistic or additive effects when we tested a combination of compound 26 and two other EZH2 inhibitors, GSK126 and EPZ6438 (Supplementary Figure 6). For GSK126, we observed $\mathrm{CI}$ values at the $\mathrm{ED}_{50}, \mathrm{ED}_{75}, \mathrm{ED}_{90}$ of $1.04,1.04$ and 1.05 in $\mathrm{BT73}$ and $\mathrm{ED}_{50}, \mathrm{ED}_{75}, \mathrm{ED}_{90}$ of $0.78,0.70$ and 0.64 in BT147. For EPZ6438, we detected CI values at the $\mathrm{ED}_{50}, \mathrm{ED}_{75}, \mathrm{ED}_{90}$ of 0.6, 0.6 and 0.61 in BT73 and $\mathrm{ED}_{50}, \mathrm{ED}_{75}, \mathrm{ED}_{90}$ of $0.6,0.75$ and 0.94 in BT147. These results suggest that simultaneously blocking EZH2 and HDAC1/2 may be a potential therapeutic avenue, as we have observed similar synergistic or additive responses with three structurally-different EZH2 inhibitors. We did not observe any additional effect on H3K27me3 or acetylation of Histone $\mathrm{H} 3$ with the combination of compound 26 and UNC1999 (Figure 7B), nor did we detect changes in total levels of EZH2, Histone H3, HDAC1 or HDAC2. There was, however, an increase in cleaved-PARP, $\gamma \mathrm{H} 2 \mathrm{AX}$, LC3BII as well as a decrease in c-MYC in cells treated with the combination of drugs, in particular in BT147 cells. These results suggest that several mechanisms are in place that can explain the synergy of the two compounds in vitro. 


\section{DISCUSSION}

Here we screened a unique epigenetic inhibitor library in order to identify candidate epigenetic modulators that play a role in GBM BTIC growth, survival and selfrenewal. To our knowledge, this is the first published report using this collection of drugs in GBM, in particular in primary human BTICs, which are greatly enriched in tumor-initiating capacity as compared to the immortalized cell lines [38]. Our results identify UNC1999 as a potent inhibitor of proliferation of human BTICs in vitro. UNC1999 is a novel SAM-competitive EZH2/

A.

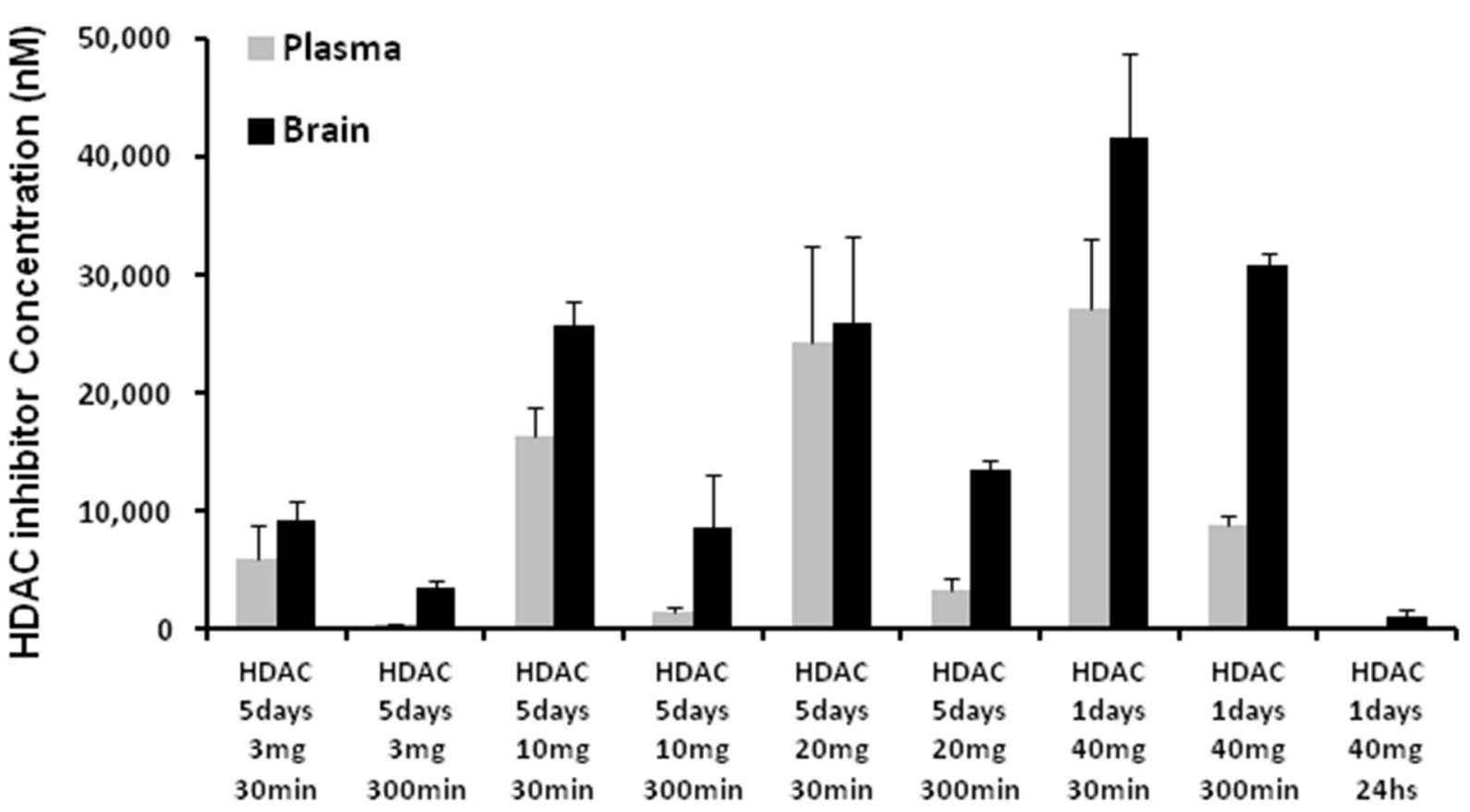

B.

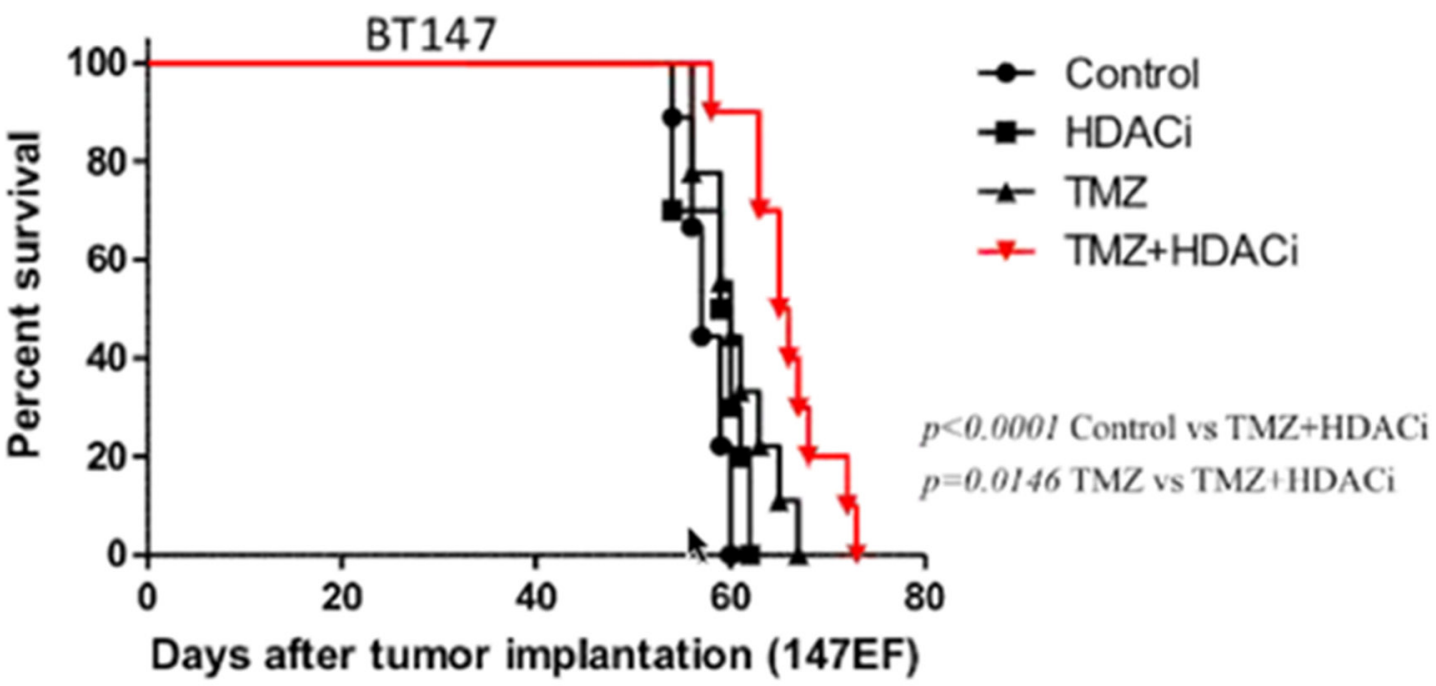

Figure 6: HDAC inhibitor compound 26 crosses the blood brain barrier and extends survival in an orthotopic tumor model in combination with TMZ. A. Pharmacokinetic results demonstrating plasma and brain concentrations in NOD/SCID mice treated with different doses of compound 26 over 5 days. B. NOD/SCID mice bearing intracranial tumors were randomized into 4 groups: 1) vehicle; 2) compound $26,10 \mathrm{mg} / \mathrm{kg}$, gavage; 3) TMZ, $30 \mathrm{mg} / \mathrm{kg}$, gavage and 4) combination of the 2 compounds. Kaplan-Meyer survival plot is shown. $(* \mathrm{p}<0.05 ; * * \mathrm{p}<0.01)$. 
EZH1 inhibitor that has demonstrated efficacy in several cancer models such as leukemia, multiple myeloma and colon cancer $[29,39,40]$. We show here that UNC1999 is cytotoxic in vitro in a diverse panel of GBM BTIC lines, inhibits self-renewal, causes cell cycle arrest at G1 stage and induces down-regulation of H3K27me3. Our data suggests that UNC1999-induced cell death is independent of either apoptosis or necroptosis, and may induce defective autophagy, as judged by the accumulation of both LC3BII and p62/SQSTM1. A similar mechanism of action has been recently described by Button et al. for dual PI3K/mTOR inhibitors [35].

A.

\section{BT73}

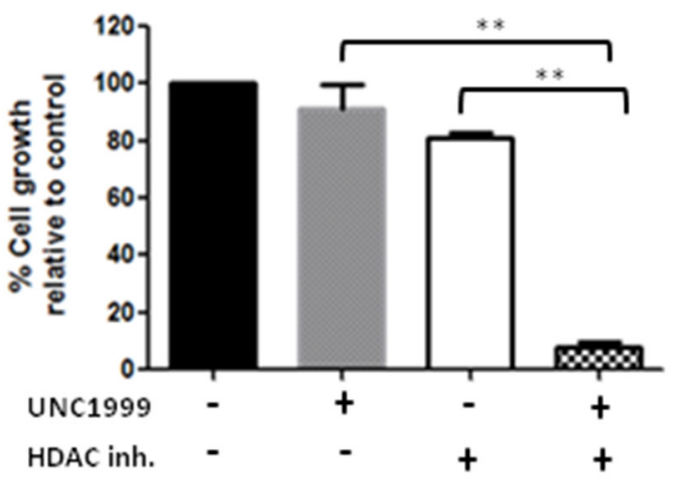

B.

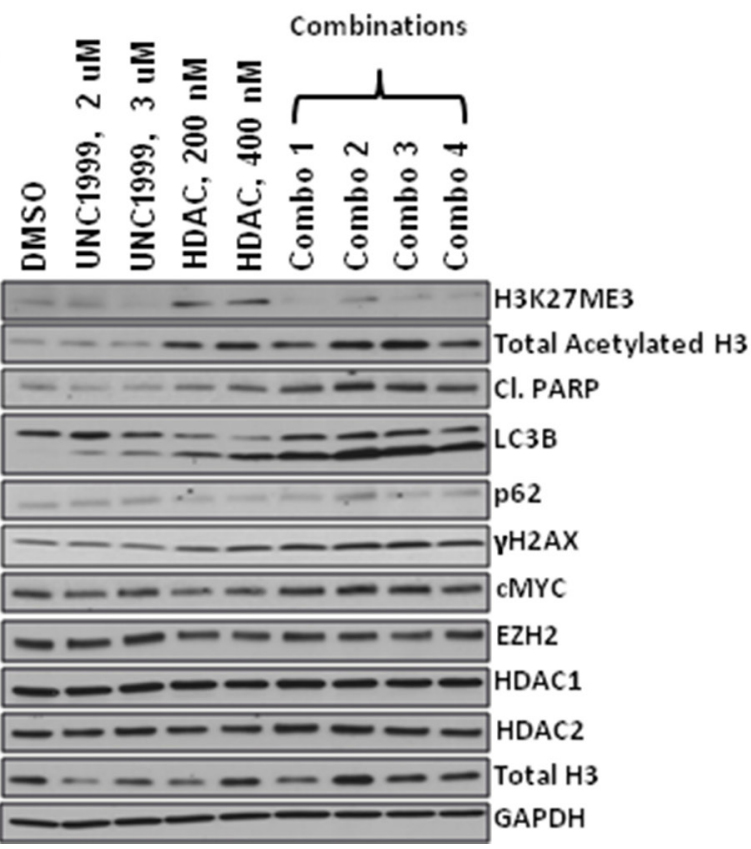

Increased and decreased expression of EZH2 have both been implicated in the development and progression of a variety of cancers types. EZH2 overexpression is associated with aggressiveness and worse prognosis in prostate, breast, bladder, endometrial tumors, melanoma and GBM [30]. Additionally, gain-of-function point mutations at Y641 of EZH2 were identified in $22 \%$ of DLBCL and 7-12\% of follicular lymphomas [41]. These mutations result in enhanced enzymatic function of EZH2 leading to increased levels of tri-methylated H3K27 and repression of PRC2 targets. However, EZH2 can also act as a tumor suppressor, with loss-of-function

\section{BT147}
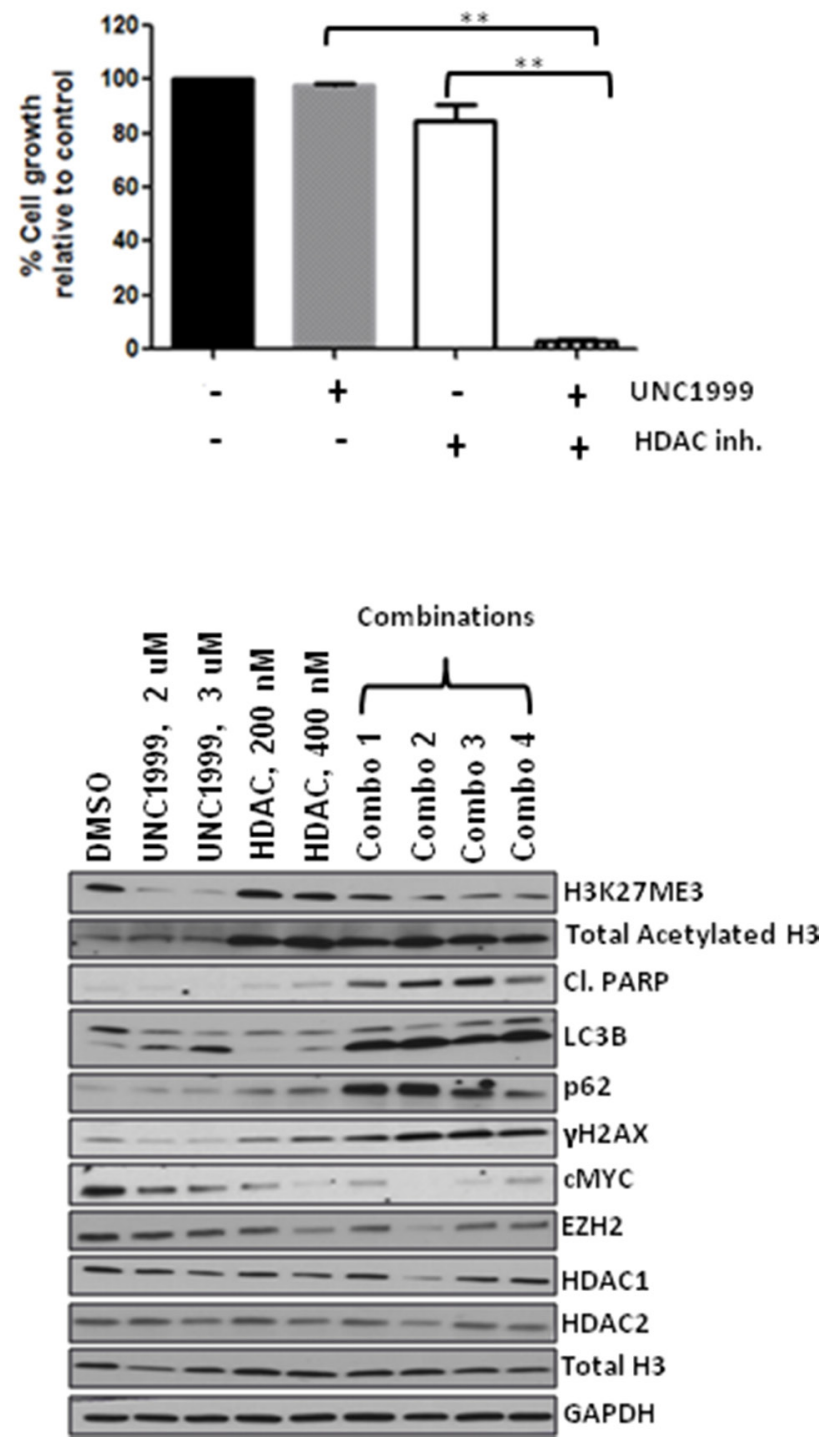

Figure 7: HDAC inhibitor compound 26 synergizes with UNC1999 in vitro through augmented apoptosis and DNA damage. A. Representative bar graphs demonstrating synergy between $\mathrm{UNC} 1999$, $3.8 \mu \mathrm{M}$ and compound 26 , $385 \mathrm{nM}$ in vitro (n=3; * $\mathrm{p}<0.05 ; * * \mathrm{p}<0.01)$. B. Representative western blots demonstrating assessment of different targets following treatment with drugs alone and a combination in vitro. 
mutations identified in a subset of myelodisplastic syndromes, myeloproliferative neoplasms and $\mathrm{T}$ cell acute lymphoblastic leukemias. Moreover, recurrent missense mutations in the PRC2 substrate lysine residue 27 of histone $\mathrm{H} 3$ and its variants occur in $31 \%$ of pediatric GBM, 78\% of diffuse intrinsic pontine glioma (DIPG) and $50 \%$ of pediatric high-grade gliomas (pHGG), but not in adult GBM. The H3K27M mutation is oncogenic, blocking growth-suppressive PRC2 methylation activity resulting in reduced $\mathrm{H} 3 \mathrm{~K} 27$ methylation and derepression of PRC2 target genes. In this regard, inhibition of H3K27 demethylation mediated by JMJD3, using the inhibitor GSK-J4, suppressed the growth of DIPG tumors grown as xenografts [24]. Thus, while EZH2 inhibition may prove beneficial in adult GBM, it will most likely be detrimental for patients with pediatric gliomas.

Until now, most of the studies in GBM have used either EZH2 knockdown or treatment with 3-deazaneplanocin (DZNep) in order to assess the effect of EZH2 inhibition [19, 42, 43]. However, DZNep is a potent S-adenosylhomocysteine inhibitor which inhibits EZH2 in an indirect manner though depletion of cellular levels of all PRC2 components, resulting in decreased H3K27 methylation. In contrast, UNC1999 directly inhibits $\mathrm{EZH} 2$ and in a potent and selective manner. Interestingly, de Vries et al. reported that prolonged EZH2 depletion in glioma switched tumor cells to a different epigenetic state that enhanced cell proliferation and DNA damage repair, resulting in tumor progression [44]. However, shortterm EZH2 depletion significantly improved survival, suggesting that determining the precise dosing schedules will be crucial for a successful implementation of EZH2 inhibitors into the clinic.

We hypothesize that the superior efficacy of UNC1999 in vitro as compared to other EZH2 inhibitors may be due to its ability to simultaneously target both EZH2 and EZH1. Konze et al. [31], which first characterized UNC1999, found that it was 10,000fold more selective for EZH2 as compared to other methyltransferases (Konze et al., Figure 2G). The only other histone methyl transferase that UNC1999 inhibited was EZH1, albeit 10-fold less potently than EZH2. In contrast, GSK126 and EPZ-6438 were over 50-fold more potent towards EZH1 than EZH2. This finding suggests that UNC1999 may be more effective in inducing BTIC cell death due to suppression of both EZH2 and EZH1.

An additional issue regarding the existing EZH2 and HDAC class I inhibitors is their inability to penetrate the blood-brain barrier (BBB) [45]. BBB penetration is one of the major issues impeding successful therapeutic targeting in GBM, as more than $98 \%$ of drugs fail to cross the BBB. Although UNC1999 did not cross the BBB, we report on the efficacy of a promising class I HDAC inhibitor, optimized for optimal brain penetration. This inhibitor was identified when a series of compounds using Entinostat as a backbone were synthesized and tested in baboons [37]. Compound 26 from these studies potently inhibited
HDAC1 and HDAC2 and crossed the BBB. This inhibitor exhibited nanomolar to low micromolar cytotoxicity in vitro, disrupted self-renewal, caused cell cycle arrest, induced apoptosis and hyper-acetylation of histone H3 and demonstrated efficacy in an orthotopic BTIC model in combination with TMZ. Interestingly, we have also found that a combination of the UNC1999 EZH2 and compound 26 HDAC class I inhibitors demonstrated synergy in vitro. This combination has been previously tested in other cancers such as gallbladder carcinoma [46], however we believe this is the first report that demonstrates such synergy in GBM.

\section{MATERIALS AND METHODS}

\section{Primary cells}

GBM BTIC lines BT50, BT53, BT67, BT73, BT75, BT92, BT100, BT119 BT143 and BT147 were previously described [14-16, 18]. BTIC lines BT69, BT140, BT161, BT167, BT194, BT198 and BT208 were cultured from a series of tumor specimens (see Supplementary Table 2) obtained following informed consent from adult GBM patients during their operative procedure as previously described [14] with the approval of the University of Calgary Ethics Review Board. Briefly, BTIC cultures were initiated in defined culture serum-free medium (SFM) and gave rise to non-adherent spheres after 7-21 days in culture. Primary tumor spheres were expanded for several passages and then cryopreserved in 10\% DMSO in SFM until use in experiments (14). All BTIC lines were used at passages of approximately 10-20.

\section{Epigenetic library}

The epigenetic library consists of 24 small molecule compounds targeting diverse epigenetic targets. The library (http://www.thesgc.org/chemical-probes) was kindly provided by the Structural Genomics Consortium (SGC, Toronto, ON; http://www.thesgc.org).

\section{Drugs}

UNC1999, GSK343, EPZ6438, GSK126 and Necrostatin were from Selleck Chemicals, Dexamethasone and Temozolomide from Sigma, Z-VAD-FMK from Calbiochem, and UNC2400, a negative control for UNC1999, was kindly provided by the SGC. The brain penetrant HDAC inhibitor Compound 26 was obtained from a six step synthesis starting from 4-bromo-2nitroaniline based on a previously reported synthetic route [37]; dx.doi.org/10.1021/cn500021p).

\section{Primary screening assay}

Five BTIC lines (BT143, BT53, BT69, BT100 and BT194) were dissociated into single cells and seeded 
at $1000-2500$ cells/well in $50 \mu \mathrm{L}$ medium in 384-well microplates. Compounds were dissolved in DMSO as $10 \mathrm{mM}$ stocks. Compound addition was performed at OICR using HP Tecan D300 digital dispenser. For each compound, 12-point, 2-fold serial dilutions were performed. Drug effects were compared to cells optimally proliferating in $0.1 \%$ DMSO alone, while wells filled with media served as background. AlamarBlue ${ }^{\circledR}(5 \mu \mathrm{L})$ was added after 3 or 6 days, and fluorescence intensity measured after $6 \mathrm{hr}$ on a PHERAstar microplate reader, equipped with a $\lambda 540$ excitation/ $\lambda 590$ emission filter.

\section{Whole genome and transcriptome sequencing}

DNA and RNA from each BTIC line and DNA from peripheral blood from the respective patients were used to construct genome and transcriptome libraries. Whole genome and transcriptome paired-end sequencing using the Illumina HiSeq2500 platform generated an average genome coverage of $40 \mathrm{X}$ and average transcriptome read counts of 200 million. Genomic sequence data from normal and tumor were then aligned to the human reference genome build GRCh37 using BWA (v0.5.7). Data from multiple lanes were merged and duplicates identified using Picard (v1.38). CNASeq (v0.0.6) and APOLLOH (v0.1.1) were used to identify copy number aberrations and loss of heterozygosity respectively. For identification of single nucleotide variants, Samtools (v1.0.2) was applied followed by filtering with MutationSeq (v1.0.2), and the results were combined with variant called with Strelka (v0.4.6.2). Small indels were also identified using Strelka. These variants were annotated using Ensembl (v69). Transcriptome reads were gap-aligned using the Jaguar pipeline (v2.0.3). Further inhouse tools were used to generate read counts for exons and genes and calculate expression levels using reads per kilobase per million mapped reads (RPKM).

\section{Western blot analysis}

For cell lysis, cells were collected, washed in cold PBS, and lysates prepared in RIPA lysis buffer. Equal amounts of protein were resolved on gradient polyacrylamide gels and subjected to immunoblotting with the following antibodies: rabbit anti-EZH2 (1:1000, CST\#5246), rabbit anti-H3K27me3 (1:1000, CST\#9733), rabbit anti-H3K27me2 (1:1000, CST\#9755), rabbit antiH3K4me1 (1:1000, CST\#5326), rabbit anti-H3K9me2 (1:1000, CST\#4658), mouse anti-H3K27ac (1:2500, Milllipore 17-683), rabbit anti-histoneH3 (1:1000, Millipore 05-928), rabbit anti-cleaved PARP (1:1000, CST\#9541L), rabbit anti-LC3B (1:1000, CST\#3868P), rabbit anti-cMYC (1:1000, CST\#13987), rabbit anti-acetyl histone H3 (1:1000, Millipore 06-599), rabbit anti-p21 (1:1000, CST\#2947S), mouse anti-HDAC1 (1:1000, CST\#5356), mouse anti-HDAC2 (1:1000, CST\#5113), rabbit anti-SQSTM1/p62 (1:1000, CST\#8025), rabbit antiphospho-Histone H2A. X (1:1000, Millipore 05-636), and rabbit anti-GAPDH (1:1000, CST\#5174). HRP-conjugated goat anti-mouse $\operatorname{IgG}(1: 5000)$ and goat anti-rabbit IgG $(1: 10,000)$ were used as the secondary antibodies.

\section{Immunofluorescence}

Cells underwent Cytospin, fixed either with 4\% PFA (anti-SQSTM1/p62) or 100\% methanol (anti-LC3B), permeabilized with $\mathrm{PBS} / 0.2 \%$ Triton $\mathrm{X}-100$, washed with PBS/ $100 \mathrm{mM}$ Glycine and then incubated in IF buffer (PBS, 0.1\% BSA, 0.2\% Triton X-100, 0.05\% Tween-20), with $2 \%$ BSA for 30 minutes as a blocking solution. Slides with cells were incubated with primary antibodies in blocking solution at $4^{\circ} \mathrm{C}$ overnight. After washing with IF buffer, the cells were incubated with secondary antibodies in blocking solution for $1 \mathrm{hr}$ at RT. Finally, after PBS washes, slides were mounted in Permount solution (Thermo, Walthman, MA). Digital image acquisition was performed with Axiovision software (Zeiss, Oberkochen, Germany) on a Zeiss Axioplan 2 microscope with a Hamamatsu (Bridgewater, NJ) Orca-R2 CCD video camera.

\section{Drug combination studies}

The combination index (CI) was used to evaluate the interaction between different drugs [47]. BT73 and BT147 were dissociated into single cells, seeded at 1000-3000 cells/well in $100 \mu \mathrm{L}$ medium in 96-well plates and treated with increasing concentrations of UNC1999, HDAC inhibitor, DEX and TMZ. Six days after the incubation, alamarBlue ${ }^{\circledR}(10 \mu \mathrm{L})$ was added to the plates and fluorescence intensity measured after $6 \mathrm{hr}$ on a PHERAstar microplate reader, equipped with a $\lambda 540$ excitation $/ \lambda 590$ emission filter. The CalcuSyn median effect model was used to calculate the CI values and evaluate whether the drug combinations were synergistic, antagonistic, or additive. CI values of $<1$ indicate synergism, $\mathrm{CI}=1$ indicate additivity, and CI $>1$ indicate antagonism [47].

\section{Pharmacokinetic (PK) properties and blood brain barrier (BBB) penetration of compounds}

To obtain plasma exposure and BBB penetration of compounds at various time points, blood and brains were collected form mouse after dosing compounds at different time points. Plasma was separated from blood by centrifugation. For analysis $20 \mu \mathrm{L}$ plasma was transferred into a $1.5 \mathrm{~mL}$ microcentrifuge tube, to which $40 \mu \mathrm{L}$ acetonitrile was added to denature and precipitate proteins, and were centrifuged for $6.5 \mathrm{~min}$. at $14000 \mathrm{rpm}$. The supernatant was transferred to LCMS vials for analysis.

For quantification of drug levels in brain samples, brains were transferred to plastic vials containing 1:2 
(brain:water, e.g. $300 \mathrm{mg}$ brain with $600 \mu \mathrm{L}$ water) volume of water and 2 glass Pyrex beads ( $4 \mathrm{~mm}$ in size). Samples were homogenized by bead beating ( 3 X 15 second pulses of 5,000 rpm) using a Precellys 24 homogenizer (Bertin Technologies, Montigny-le-Bretonneux, France). Each sample was mixed vigorously with 2 volumes of acetonitrile to precipitate out protein and were centrifuged for $6.5 \mathrm{~min}$. at $14000 \mathrm{rpm}$. The supernatant was then transferred to LCMS vials for analysis. The concentration in brain was corrected for dilution.

\section{LCMS analysis}

For each analysis, a calibration curve with ten different concentrations starting from $10 \mathrm{mg} / \mathrm{mL}$ up to $10,000 \mathrm{ng} / \mathrm{mL}$ was prepared by spiking blank plasma. Chromatographic separations were carried out on an Acquity UPLC BEH C18 (2.1 X 50 mm, $1.7 \mu \mathrm{m})$ column using ACQUITY UPLC II system. The mobile phase was $0.1 \%$ formic acid in water (solvent $\mathrm{A}$ ) and $0.1 \%$ formic acid in acetonitrile (solvent B). A gradient starting at 95\% solvent A going to 5\% in 4.5 min., holding for 0.5 min., going back to $95 \%$ in $0.5 \mathrm{~min}$. and equilibrating the column for 1 min. was employed. A Waters Synapt G2S QTof mass spectrometer equipped with an atmospheric pressure ionization source was used for mass spectrometric analysis. MassLynx 4.1 was used for data analysis.

\section{Determination of cellular DNA content}

The BTIC line BT73 $\left(0.2 \times 10^{6}\right)$ was plated in 6-well dishes and DMSO, UNC1999 $(2 \mu \mathrm{M}, 3 \mu \mathrm{M}$ and $5 \mu \mathrm{M})$ or HDAC inhibitor $(0.2 \mu \mathrm{M}, 0.4 \mu \mathrm{M}, 0.6 \mu \mathrm{M}$ and $1 \mu \mathrm{M})$ added. After 48 hrs (UNC1999) or 72 hrs (HDAC inhibitor), cells were harvested, washed with PBS and fixed in 70\% ice-cold ethanol. Fixed cells were treated with RNAseA, stained with Propidium Iodide at $37^{\circ} \mathrm{C}$ for $1 \mathrm{hr}$ and analyzed on a LSR II flow cytometer to determine cellular DNA content.

\section{Viable cell counts following treatment with UNC1999 and HDAC inhibitor}

$1 \times 10^{5}$ dissociated BTICs (BT73) were seeded in triplicate in 12-well non-tissue culture-treated plates in $2 \mathrm{~mL}$ of medium. UNC1999 $(4 \mu \mathrm{M}-6 \mu \mathrm{M})$, HDAC inhibitor $(0.1 \mu \mathrm{M}-0.4 \mu \mathrm{M})$ or $0.1 \%$ DMSO were added to the cells $24 \mathrm{hrs}$ later. At specific time points, spheres were collected, dissociated and subjected to a viable cell count by trypan blue exclusion.

\section{Annexin $\mathrm{V}$ assay}

The ability of drugs to induce apoptosis in BTICs was determined with an Annexin V-FITC detection kit, used according to the manufacturer's instructions (BD

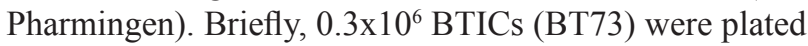
in 6-well dishes and treated with various concentrations of UNC1999 (3-6 $\mu \mathrm{M})$ or HDAC inhibitor $(0.4-1$ $\mu \mathrm{M})$. Cells were harvested 24-72 hr later, stained for Annexin V/propidium iodide and analyzed on a LSR II flow cytometer. Relative numbers of early apoptotic cells (Annexin V-positive, Propidium iodide-negative) and late apoptotic cells (Annexin V-positive, Propidium iodidepositive) were obtained for each time point.

\section{Sphere-formation assay}

BTICs (BT73 and BT147) were seeded in triplicate in non-TC-treated 96-well microplates at a density of 1000-2500 cells/well in $50 \mu \mathrm{L} /$ well. Compounds were diluted in medium $(1: 1000)$ and immediately added to the cells in a volume of $50 \mu \mathrm{L}$ (final concentration of DMSO $=0.05 \%$ ). Cells were re-treated $72 \mathrm{hrs}$ post-plating with drugs, and fixed after 6 days with 4\% paraformaldehyde (Electron Microscopy Sciences). Sphere number was determined by manual counting, and the results expressed as the mean sphere number of treated wells as compared to DMSO-treated wells*100.

\section{Flank xenograft model}

$1.5 \times 10^{6}$ BTICs $(73 \mathrm{M})$ were resuspended in media and injected in $100 \mu \mathrm{l}$ volume subcutaneously into 6-8week old NOD/SCID mice. Drug treatment began when tumor size reached $\sim 25 \mathrm{~mm}^{3}$. Animals were randomized into 4 groups: 1) Vehicle; 2) UNC1999 alone; 3) DEX alone; 4) UNC1999 + DEX. Mice were injected via oral gavage with either vehicle (10\% DMSO, 40\% PEG) or UNC1999 (150 mg/kg) every day for 17 days. An additional group of mice received intraperitoneal injection of DEX ( $1 \mathrm{mg} / \mathrm{kg})$ every day for 17 days. The combination group received both UNC1999 and DEX administered by oral gavage and i.p. injection respectively.

\section{Orthotopic xenograft model}

BT147 spheres were dissociated into single cell suspensions and $1 \times 10^{5}$ cells were stereotactically implanted into the right striata of 6-8 week old NOD/SCID mice as previously described [14]. Seven days after BTIC implantation, mice were randomized into 4 groups and injected via oral gavage for a total of 3 cycles ( 5 days ON, 2 days OFF): 1) vehicle (50\% PEG, 50\% water); 2) HDAC inhibitor alone (10 mg/kg); 3) Temozolomide (TMZ) alone $(30 \mathrm{mg} / \mathrm{kg})$ and 4) combination of HDAC inhibitor and Temozolomide.

\section{Statistical analysis}

Statistical analyses were performed with Microsoft Excel using an unpaired, two-tailed student's T-test with $\mathrm{P}<0.05$ as the significance cutoff. One-way ANOVA was used to determine statistically significant differences from the mean in the combination study in vivo. 


\section{Abbreviations}

GBM, Glioblastoma; BTICs, Brain tumor-initiating cells; SGC, Structural Genomics Consortium; BBB, Blood-brain-barrier; TMZ, Temozolomide; MGMT, O-6-methylguanine-DNA methyltransferase; DEX, Dexamethasone; NOD/SCID, Non-obese diabetic/severe combined immune deficiency; SKPs, Skin-derived precursor cells, EZH2, Enhancer of zeste homologue 2; HDAC, Histone deacetylase; SAM, S-adenosyl methionine; PRC2, Polycomb repressive complex 2; CI, combination index; DZNep, 3-deazaneplanocin.

\section{ACKNOWLEDGMENTS}

We thank Peter Brown and Cheryl Arrowsmith from SGC for providing the epigenetic library and the ARC UHN facility for assistance with animal studies. We also thank members of the TFRI "Therapeutic Targeting of GBM" team for their contributions of reagents and comments for this study.

\section{CONFLICTS OF INTEREST}

The authors declare no conflicts of interest.

\section{GRANT SUPPORT}

This work was supported by grants from the Terry Fox Research Institute and the Canadian Stem Cell Network. D.R.K. holds a Tier I Canada Research Chair. M.A.M. acknowledges the BC Cancer Agency, the BC Cancer Foundation, the Canada Foundation for Innovation, the Canada Research Chairs program, Genome British Columbia and the Canadian Institutes of Health Research (CIHR-262119 and FDN-143288).

\section{REFERENCES}

1. Stupp R, Mason WP, van den Bent MJ, Weller M, Fisher B, Taphoorn MJ, Belanger K, Brandes AA, Marosi C, Bogdahn U, Curschmann J, Janzer RC, Ludwin SK, et al. Radiotherapy plus concomitant and adjuvant temozolomide for glioblastoma. N Engl J Med. 2005; 352:987-996.

2. Meyer M, Reimand J, Lan X, Head R, Zhu X, Kushida M, Bayani J, Pressey JC, Lionel AC, Clarke ID, Cusimano M, Squire JA, Scherer SW, et al. Single cell-derived clonal analysis of human glioblastoma links functional and genomic heterogeneity. Proc Natl Acad Sci U S A. 2015; 112:851-856.

3. Patel AP, Tirosh I, Trombetta JJ, Shalek AK, Gillespie SM, Wakimoto H, Cahill DP, Nahed BV, Curry WT, Martuza RL, Louis DN, Rozenblatt-Rosen O, Suva ML, et al. Single-cell
RNA-seq highlights intratumoral heterogeneity in primary glioblastoma. Science. 2014; 344:1396-1401.

4. Pardridge WM. The blood-brain barrier: bottleneck in brain drug development. NeuroRx. 2005; 2:3-14.

5. Bao S, Wu Q, McLendon RE, Hao Y, Shi Q, Hjelmeland AB, Dewhirst MW, Bigner DD, Rich JN. Glioma stem cells promote radioresistance by preferential activation of the DNA damage response. Nature. 2006; 444:756-760.

6. Chen J, Li Y, Yu TS, McKay RM, Burns DK, Kernie SG, Parada LF. A restricted cell population propagates glioblastoma growth after chemotherapy. Nature. 2012; 488:522-526.

7. Singh SK, Hawkins C, Clarke ID, Squire JA, Bayani J, Hide T, Henkelman RM, Cusimano MD, Dirks PB. Identification of human brain tumour initiating cells. Nature. 2004; 432:396-401.

8. Kondo Y, Katsushima K, Ohka F, Natsume A, Shinjo K. Epigenetic dysregulation in glioma. Cancer Sci. 2014; 105:363-369.

9. Nagarajan RP, Costello JF. Epigenetic mechanisms in glioblastoma multiforme. Semin Cancer Biol. 2009; 19:188-197.

10. Hegi ME, Diserens AC, Gorlia T, Hamou MF, de Tribolet N, Weller M, Kros JM, Hainfellner JA, Mason W, Mariani L, Bromberg JE, Hau P, Mirimanoff RO, Cairncross JG, Janzer RC, Stupp R. MGMT gene silencing and benefit from temozolomide in glioblastoma. N Engl J Med. 2005; 352:997-1003.

11. Brennan CW, Verhaak RG, McKenna A, Campos B, Noushmehr H, Salama SR, Zheng S, Chakravarty D, Sanborn JZ, Berman SH, Beroukhim R, Bernard B, Wu CJ, Genovese G, Shmulevich I, Barnholtz-Sloan J, et al. The somatic genomic landscape of glioblastoma. Cell. 2013; 155:462-477.

12. Blough MD, Westgate MR, Beauchamp D, Kelly JJ, Stechishin O, Ramirez AL, Weiss S, Cairncross JG. Sensitivity to temozolomide in brain tumor initiating cells. Neuro Oncol. 2010; 12:756-760.

13. Kelly JJ, Blough MD, Stechishin OD, Chan JA, Beauchamp D, Perizzolo M, Demetrick DJ, Steele L, Auer RN, Hader WJ, Westgate M, Parney IF, Jenkins R, Cairncross JG, Weiss S. Oligodendroglioma cell lines containing $\mathrm{t}(1 ; 19)$ (q10;p10). Neuro Oncol. 2010; 12:745-755.

14. Kelly JJ, Stechishin O, Chojnacki A, Lun X, Sun B, Senger DL, Forsyth P, Auer RN, Dunn JF, Cairncross JG, Parney IF, Weiss S. Proliferation of human glioblastoma stem cells occurs independently of exogenous mitogens. Stem Cells. 2009; 27:1722-1733.

15. Luchman HA, Stechishin OD, Dang NH, Blough MD, Chesnelong C, Kelly JJ, Nguyen SA, Chan JA, Weljie AM, Cairncross JG, Weiss S. An in vivo patient-derived model of endogenous IDH1-mutant glioma. Neuro Oncol. 2012; 14:184-191. 
16. Luchman HA, Stechishin OD, Nguyen SA, Lun XQ, Cairncross JG, Weiss S. Dual mTORC1/2 blockade inhibits glioblastoma brain tumor initiating cells in vitro and in vivo and synergizes with temozolomide to increase orthotopic xenograft survival. Clin Cancer Res. 2014; 20:5756-5767.

17. Lun X, Wells JC, Grinshtein N, King JC, Hao X, Dang NH, Wang X, Aman A, Uehling D, Datti A, Wrana JL, Easaw JC, Luchman A, et al. Disulfiram when Combined with Copper Enhances the Therapeutic Effects of Temozolomide for the Treatment of Glioblastoma. Clin Cancer Res. 2016 Mar 22. [Epub ahead of print].

18. Nguyen SA, Stechishin OD, Luchman HA, Lun XQ, Senger DL, Robbins SM, Cairncross G, Weiss S. Novel MSH6 mutations in treatment naive glioblastoma and anaplastic oligodendroglioma influence temozolomide resistance independently of MGMT methylation. Clin Cancer Res. 2014.

19. Zhang J, Chen L, Han L, Shi Z, Pu P, Kang C. EZH2 is a negative prognostic factor and exhibits pro-oncogenic activity in glioblastoma. Cancer Lett. 2015; 356:929-936.

20. Lee P, Murphy B, Miller R, Menon V, Banik NL, Giglio P, Lindhorst SM, Varma AK, Vandergrift WA, 3rd, Patel SJ, Das A. Mechanisms and clinical significance of histone deacetylase inhibitors: epigenetic glioblastoma therapy. Anticancer Res. 2015; 35:615-625.

21. Li G, Warden C, Zou Z, Neman J, Krueger JS, Jain A, Jandial R, Chen M. Altered expression of polycomb group genes in glioblastoma multiforme. PLoS One. 2013; 8:e80970.

22. Bezecny P. Histone deacetylase inhibitors in glioblastoma: pre-clinical and clinical experience. Med Oncol. 2014; 31:985.

23. Orzan F, Pellegatta S, Poliani PL, Pisati F, Caldera V, Menghi F, Kapetis D, Marras C, Schiffer D, Finocchiaro G. Enhancer of Zeste 2 (EZH2) is up-regulated in malignant gliomas and in glioma stem-like cells. Neuropathol Appl Neurobiol. 2011; 37:381-394.

24. Hashizume R, Andor N, Ihara Y, Lerner R, Gan H, Chen X, Fang D, Huang X, Tom MW, Ngo V, Solomon D, Mueller $\mathrm{S}$, Paris PL, et al. Pharmacologic inhibition of histone demethylation as a therapy for pediatric brainstem glioma. Nat Med. 2014; 20:1394-1396.

25. Cheng Z, Gong Y, Ma Y, Lu K, Lu X, Pierce LA, Thompson RC, Muller S, Knapp S, Wang J. Inhibition of BET bromodomain targets genetically diverse glioblastoma. Clin Cancer Res. 2013; 19:1748-1759.

26. Gallo M, Coutinho FJ, Vanner RJ, Gayden T, Mack SC, Murison A, Remke M, Li R, Takayama N, Desai K, Lee L, Lan X, Park NI, et al. MLL5 Orchestrates a Cancer SelfRenewal State by Repressing the Histone Variant H3.3 and Globally Reorganizing Chromatin. Cancer Cell. 2015.

27. Eyupoglu IY, Hahnen E, Trankle C, Savaskan NE, Siebzehnrubl FA, Buslei R, Lemke D, Wick W, Fahlbusch R, Blumcke I. Experimental therapy of malignant gliomas using the inhibitor of histone deacetylase MS-275. Mol Cancer Ther. 2006; 5:1248-1255.
28. Herviou L, Cavalli G, Cartron G, Klein B, Moreaux J. EZH2 in normal hematopoiesis and hematological malignancies. Oncotarget. 2015. doi: 10.18632/oncotarget.6198.

29. Xu B, On DM, Ma A, Parton T, Konze KD, Pattenden SG, Allison DF, Cai L, Rockowitz S, Liu S, Liu Y, Li F, Vedadi $\mathrm{M}$, et al. Selective inhibition of EZH2 and EZH1 enzymatic activity by a small molecule suppresses MLL-rearranged leukemia. Blood. 2015; 125:346-357.

30. Kim KH, Roberts CW. Targeting EZH2 in cancer. Nat Med. 2016; 22:128-134.

31. Konze KD, Ma A, Li F, Barsyte-Lovejoy D, Parton T, Macnevin CJ, Liu F, Gao C, Huang XP, Kuznetsova E, Rougie M, Jiang A, Pattenden SG, et al. An orally bioavailable chemical probe of the Lysine Methyltransferases EZH2 and EZH1. ACS Chem Biol. 2013; 8:1324-1334.

32. Liu TP, Lo HL, Wei LS, Hsiao HH, Yang PM. S-AdenosylL-methionine-competitive inhibitors of the histone methyltransferase EZH2 induce autophagy and enhance drug sensitivity in cancer cells. Anticancer Drugs. 2015; 26:139-147.

33. Melo-Lima S, Celeste Lopes M, Mollinedo F. Necroptosis is associated with low procaspase-8 and active RIPK1 and -3 in human glioma cells. Oncoscience. 2014; 1:649-664. doi: 10.18632/oncoscience.89.

34. Knutson SK, Warholic NM, Johnston LD, Klaus CR, Wigle TJ, Iwanowicz D, Littlefield BA, Porter-Scott M, Smith JJ, Moyer MP, Copeland RA, Pollock RM, Kuntz KW, et al. Synergistic Anti-Tumor Activity of EZH2 Inhibitors and Glucocorticoid Receptor Agonists in Models of Germinal Center Non-Hodgkin Lymphomas. PLoS One. 2014; 9:e111840.

35. Button RW, Vincent JH, Strang CJ, Luo S. Dual PI-3 kinase/ mTOR inhibition impairs autophagy flux and induces cell death independent of apoptosis and necroptosis. Oncotarget. 2016; 7:5157-5175. doi: 10.18632/oncotarget.6986.

36. Nakamura M, Kikukawa Y, Takeya M, Mitsuya H, Hata H. Clarithromycin attenuates autophagy in myeloma cells. Int J Oncol. 2010; 37:815-820.

37. Seo YJ, Kang Y, Muench L, Reid A, Caesar S, Jean L, Wagner F, Holson E, Haggarty SJ, Weiss P, King P, Carter P, Volkow ND, Fowler JS, Hooker JM, Kim SW. Image-guided synthesis reveals potent blood-brain barrier permeable histone deacetylase inhibitors. ACS Chem Neurosci. 2014; 5:588-596.

38. Lee J, Kotliarova S, Kotliarov Y, Li A, Su Q, Donin NM, Pastorino S, Purow BW, Christopher N, Zhang W, Park JK, Fine HA. Tumor stem cells derived from glioblastomas cultured in bFGF and EGF more closely mirror the phenotype and genotype of primary tumors than do serumcultured cell lines. Cancer Cell. 2006; 9:391-403.

39. Agarwal P, Alzrigat M, Parraga AA, Enroth S, Singh U, Ungerstedt J, Osterborg A, Brown PJ, Ma A, Jin J, Nilsson K, Oberg F, Kalushkova A, Jernberg-Wiklund H. Genome-wide profiling of histone H3 lysine 27 and lysine 4 trimethylation in multiple myeloma reveals the importance 
of Polycomb gene targeting and highlights EZH2 as a potential therapeutic target. Oncotarget. 2016; 7:6809-6823. doi: 10.18632/oncotarget.6843.

40. Katona BW, Liu Y, Ma A, Jin J, Hua X. EZH2 inhibition enhances the efficacy of an EGFR inhibitor in suppressing colon cancer cells. Cancer Biol Ther. 2014; 15:1677-1687.

41. Morin RD, Johnson NA, Severson TM, Mungall AJ, An J, Goya R, Paul JE, Boyle M, Woolcock BW, Kuchenbauer F, Yap D, Humphries RK, Griffith OL, et al. Somatic mutations altering EZH2 (Tyr641) in follicular and diffuse large B-cell lymphomas of germinal-center origin. Nature Genetics. 2010; 42:181-185.

42. Suva ML, Riggi N, Janiszewska M, Radovanovic I, Provero P, Stehle JC, Baumer K, Le Bitoux MA, Marino D, Cironi L, Marquez VE, Clement V, Stamenkovic I. EZH2 is essential for glioblastoma cancer stem cell maintenance. Cancer Res. 2009; 69:9211-9218.

43. Kim E, Kim M, Woo DH, Shin Y, Shin J, Chang N, Oh YT, Kim H, Rheey J, Nakano I, Lee C, Joo KM, Rich JN, Nam DH, Lee J. Phosphorylation of EZH2 activates
STAT3 signaling via STAT3 methylation and promotes tumorigenicity of glioblastoma stem-like cells. Cancer Cell. 2013; 23:839-852.

44. de Vries NA, Hulsman D, Akhtar W, de Jong J, Miles DC, Blom M, van Tellingen O, Jonkers J, van Lohuizen M. Prolonged Ezh2 Depletion in Glioblastoma Causes a Robust Switch in Cell Fate Resulting in Tumor Progression. Cell Rep. 2015; S2211-1247(14)01057-2.

45. Zhang P, de Gooijer MC, Buil LC, Beijnen JH, Li G, van Tellingen $\mathrm{O} . \mathrm{ABCB} 1$ and $\mathrm{ABCG} 2$ restrict the brain penetration of a panel of novel EZH2-Inhibitors. Int J Cancer. 2015; 137:2007-2018.

46. Yamaguchi J, Sasaki M, Sato Y, Itatsu K, Harada K, Zen Y, Ikeda H, Nimura Y, Nagino M, Nakanuma Y. Histone deacetylase inhibitor (SAHA) and repression of EZH2 synergistically inhibit proliferation of gallbladder carcinoma. Cancer Sci. 2010; 101:355-362.

47. Chou TC, Talalay P. Quantitative analysis of dose-effect relationships: the combined effects of multiple drugs or enzyme inhibitors. Adv Enzyme Regul. 1984; 22:27-55. 